\title{
Paradoxical Excitatory Impact of SK Channels on Dendritic Excitability
}

\author{
자 Tobias Bock, Suraj Honnuraiah, and Greg J. Stuart \\ Eccles Institute of Neuroscience and Australian Research Council Centre of Excellence for Integrative Brain Function, John Curtin School of Medical \\ Research, Australian National University, Canberra, 2601 Australia
}

Dendritic excitability regulates how neurons integrate synaptic inputs and thereby influences neuronal output. As active dendritic events are associated with significant calcium influx they are likely to be modulated by calcium-dependent processes, such as calcium-activated potassium channels. Here we investigate the impact of small conductance calcium-activated potassium channels (SK channels) on dendritic excitability in male and female rat cortical pyramidal neurons in vitro and in vivo. Using local applications of the SK channel antagonist apamin in vitro, we show that blocking somatic SK channels enhances action potential output, whereas blocking dendritic SK channels paradoxically reduces the generation of dendritic calcium spikes and associated somatic burst firing. Opposite effects were observed using the SK channel enhancer NS309. The effect of apamin on dendritic SK channels was occluded when R-type calcium channels were blocked, indicating that the inhibitory impact of apamin on dendritic calcium spikes involved R-type calcium channels. Comparable effects were observed in vivo. Intracellular application of apamin via the somatic whole-cell recording pipette reduced the medium afterhyperpolarization and increased action potential output during UP states. In contrast, extracellular application of apamin to the cortical surface to block dendritic SK channels shifted the distribution of action potentials within UP states from an initial burst to a more distributed firing pattern, while having no impact on overall action potential firing frequency or UP and DOWN states. These data indicate that somatic and dendritic SK channels have opposite effects on neuronal excitability, with dendritic SK channels counterintuitively promoting rather than suppressing neuronal output.

Key words: calcium; cortex; dendrite; excitability; pyramidal neuron; SK channel

\section{Significance Statement}

Neurons typically receive input from other neurons onto processes called dendrites, and use electrical events such as action potentials for signaling. As electrical events in neurons are usually associated with calcium influx they can be regulated by calcium-dependent processes. One such process is through the activation of calcium-dependent potassium channels, which usually act to reduce action potential signaling. Although this is the case for calcium-dependent potassium channels found at the cell body, we show here that calcium-dependent potassium channels in dendrites of cortical pyramidal neurons counterintuitively promote rather than suppress action potential output.

\section{Introduction}

Pyramidal neurons in layer 5 of the neocortex are considered to be the main output pathway for the transfer of cortical signals to other brain regions. Evidence from over 20 years ago indicates

Received Jan. 13, 2019; revised Aug. 7, 2019; accepted Aug. 12, 2019.

Author contributions: T.B. and G.J.S. designed research; T.B. and S.H. performed research; T.B. analyzed data; T.B. and G.J.S. wrote the paper.

This work was supported by the National Health and Medical Research Council of Australia as well as the Australian Research Council Centre of Excellence for Integrative Brain Function.

The authors declare no competing financial interests.

Correspondence should be addressed to Tobias Bock at htb2110@columbia.edu or Greg J. Stuart at greg.stuart@anu.edu.au.

T. Bock's present address: Jerome L. Greene Science Center, New York, NY 10027.

https://doi.org/10.1523/JNEUROSCI.0105-19.2019

Copyright $\odot 2019$ the authors that the generation of action potentials (APs) in these neurons is influenced by active signal processing in their dendrites (Amitai et al., 1993; Regehr et al., 1993). A variety of regenerative dendritic events have since been described in layer 5 pyramidal neurons, including backpropagating action potentials (Stuart and Sakmann, 1994) as well as dendritic sodium (Stuart et al., 1997), calcium (Schiller et al., 1997; Larkum et al., 1999b) and NMDA spikes (Schiller et al., 2000). These active dendritic events can shift neuronal output in cortical pyramidal neurons from regular AP firing during somatic and proximal dendritic depolarization to burst firing during more distal dendritic excitatory input (Larkum et al., 1999b; Williams and Stuart, 1999), which has recently been linked to modulating sensory perception threshold (Manita et al., 2015; Takahashi et al., 2016). Similar observations have 
been made in hippocampal pyramidal neurons (Golding et al., 1999; Magee and Carruth, 1999), where dendritic spikes have also been identified as an essential component in the emergence of place fields (Bittner et al., 2015). Because active dendritic events are either mediated or accompanied by activation of voltagegated calcium channels, they are associated with an increase in dendritic intracellular calcium. As a result calcium-activated potassium channels are likely to play a key role in regulating dendritic excitability and its associated impact on neuronal output.

Consistent with this idea, previous work indicates that large conductance calcium-activated potassium channels (BK channels) can modulate the occurrence and duration of dendritic calcium spikes in both cortical and hippocampal pyramidal neurons (Golding et al., 1999; Benhassine and Berger, 2009; Bock and Stuart, 2016b). Although less is known about the impact of small conductance calcium-activated potassium channels (SK channels) on dendritic excitability, they have been shown to regulate the duration of dendritic plateau potentials induced by glutamate uncaging in hippocampal neurons (Cai et al., 2004) as well as the amplitude and threshold of NMDA spikes in cortical pyramidal neurons (Bock and Stuart, 2016b). To date, the impact of SK channels on dendritic calcium spikes in pyramidal neurons has not been investigated. At the soma, SK channels contribute to the generation of the medium afterhyperpolarization (mAHP) and thereby regulate the frequency of AP firing in cortical (Schwindt et al., 1988b) and hippocampal pyramidal neurons (Sah and McLachlan, 1992; but see Gu et al., 2008). SK channels are also expressed in the dendrites and spines of pyramidal neurons, where they regulate NMDA receptor activation during EPSPs (Faber et al., 2005; Ngo-Anh et al., 2005; Bloodgood and Sabatini, 2007), calcium influx during backpropagating APs (Jones and Stuart, 2013) and have recently been shown to regulate expression of spike timing-dependent plasticity (Jones et al., 2017). In this study we examine the impact of SK channels on dendritic excitability in cortical layer 5 pyramidal neurons both in vitro and in vivo. Paradoxically, we find that dendritic SK channels in these neurons enhance dendritic excitability leading to an increase in neuronal output.

\section{Materials and Methods}

Slice preparation. Wistar rats (4-6 weeks old) of either sex were anesthetized by inhalation of isoflurane (2\%) and decapitated according to the procedures approved by the Animal Ethics Committee of the Australian National University. The skull was opened and the brain removed and immediately transferred into ice-cold carbogenated artificial CSF [(ACSF); in mM: $125 \mathrm{NaCl}, 2 \mathrm{CaCl}_{2}, 1 \mathrm{MgCl}_{2}, 25 \mathrm{NaHCO}_{3}, 3 \mathrm{KCl}, 1.25$ $\mathrm{NaH}_{2} \mathrm{PO}_{4}, \mathrm{pH}$ 7.2]. During the slicing procedure, the $\mathrm{MgCl}_{2}$ concentration was increased to $5 \mathrm{~mm}$ to reduce cell excitability. The brain was cut along the midline, adhered to an angled $\left(10-15^{\circ}\right)$ slicing platform and immediately re-submerged. Three hundred-micrometer-thick brain slices were cut using a vibrating tissue slicer (Campton or Leica Microsystems) and transferred to a chamber containing ACSF at $35^{\circ} \mathrm{C}$, where they were incubated for $40 \mathrm{~min}$. Thereafter, they were held at room temperature $\left(21^{\circ} \mathrm{C}\right)$ until required.

Electrophysiology. All in vitro electrophysiological recordings were performed using an Olympus BX61 WI microscope, equipped with Dodt gradient contrast optics (Luigs and Neumann) and a fluorescent imaging system. Slices were continuously perfused with carbogenated ACSF at a rate of $2 \mathrm{ml} / \mathrm{min}$ at $34 \pm 1^{\circ} \mathrm{C}$. Borosilicate glass pipettes (inner diameter $0.5 \mathrm{~mm}$, outer diameter $1 \mathrm{~mm}$ ) were pulled by a computer controlled electrode puller (Sutter Instruments) and had open tip resistances of 4-6 $\mathrm{M} \Omega$ for somatic recording pipettes and $9-12 \mathrm{M} \Omega$ for dendritic recording pipettes. Recording pipettes were filled with intracellular solution of the following composition (in $\mathrm{mM}$ ): $10 \mathrm{KCl}, 130 \mathrm{~K}$-Gluconate, $10 \mathrm{HEPES}, 4$ Mg-ATP, $0.3 \mathrm{Na}_{2}$-GTP, $10 \mathrm{Na}_{2}$-phosphocreatine, $\mathrm{pH}$ set to 7.25 with $\mathrm{KOH}$, osmolarity $285 \mathrm{mOsm} / \mathrm{L}$. Patch pipettes were electrically connected via a chlorided silver wire to voltage- and current-clamp amplifiers via amplifier head stages, which were mounted on remotely controlled micromanipulators (Luigs and Neumann). Voltage and current-clamp recordings were obtained with BVC-700A amplifiers (Dagan). Electrophysiological data were filtered at $10 \mathrm{kHz}$ and acquired at 50 $\mathrm{kHz}$ by a Macintosh computer running AxoGraph X acquisition software (AxoGraph Scientific) using an ITC-18 interface (InstruTECH, HEKA).

In recordings from the main apical dendrite, dendritic whole-cell patch-clamp recordings were established $600-800 \mu \mathrm{m}$ from the soma and not more than $100 \mu \mathrm{m}$ from the first bifurcation. During dendritic recordings from apical tuft branches the dendritic recording site was up to $300 \mu \mathrm{m}$ distal to the initial apical bifurcation on secondary or tertiary branches. A somatic whole-cell patch-clamp recording was first established and the cell was filled with the fluorescent dye AlexaFluor 594 (5 $\mu \mathrm{M}$; Invitrogen), which was added to the intracellular solution of the somatic patch pipette. After allowing time for the dye to perfuse into the cell a fluorescent image of the dendritic tree was overlaid on top of the Dodt gradient contrast image using customized software (Dancam) to coordinate a pair of shutters (Sutter Instruments) located before the transmitted and epifluorescent light paths. All images were acquired with a CoolSNAP EZ CCD camera (Photometrics). If electrode or tissue drift was observed during the course of the experiment the electrode position was adjusted to maintain close proximity to the soma or dendrite. In some cases, slight positive pressure was applied (not $>3 \mathrm{mmHg}$ ) to prevent clogging of the pipette tip. Cells were excluded from data analysis if the somatic resting membrane potential was more depolarized than -55 $\mathrm{mV}$ or if fluctuations in membrane potential $>5 \mathrm{mV}$ were observed at any time during the recording. Membrane potentials were not corrected for the liquid junction potential between the pipette and bath solution, which was $\sim 13 \mathrm{mV}$.

Cells were also excluded if the somatic series resistance exceeded 20 $\mathrm{M} \Omega$ or if the somatic series resistance changed by $>10 \%$ during the recording. Dendritic recordings were discarded if the dendritic series resistance exceeded $40 \mathrm{M} \Omega$ or if it changed by $>15 \%$.

Pharmacology. Drugs were either washed in via the bath solution or applied locally using a glass pipette. The tip of this application pipette was broken under visual control to obtain a diameter of $20-30 \mu \mathrm{m}$. This pipette was positioned just above the surface of the slice and drugs were locally applied using $20-30 \mathrm{mmHg}$ of positive pressure. Local applications were always made first to the dendritic recording location and then to the somatic recording location. The area of the slice perfused during local application of drugs was estimated to be $\sim 150 \mu \mathrm{m}$ in diameter based on spread of the fluorescent dye AlexaFluor 594. Local applications of ACSF alone caused no change in the response of cells during somatic and dendritic current injections, indicating that this application system does not cause artifacts due to tissue movement or other unintended side effects (data not shown).

Apamin (Tocris Bioscience) was dissolved in purified water to a stock concentration of $500 \mu \mathrm{M}$ and stored at $-20^{\circ} \mathrm{C}$ until required. On the day of the experiment apamin was diluted in ACSF to a concentration of 100 $\mathrm{nm}$ for bath application or $1 \mu \mathrm{M}$ for local application. The alternative SK channel blocker UCL1684 (Tocris Bioscience) and the SK channel enhancer NS309 (Tocris Bioscience) were dissolved in dimethyl-sulfoxide to a stock concentration of $100 \mathrm{~mm}$ and stored at $-20^{\circ} \mathrm{C}$ until required. On the day of the experiment UCL1684 and NS309 were diluted in ACSF to a concentration of $100 \mu \mathrm{M}$ for local application. To block potential presynaptic effects of apamin, in some in vitro experiments glutamatergic and GABAergic transmission were blocked by bath application of DNQX (10 $\mu \mathrm{M}$; Tocris Bioscience), D-AP5 (100 $\mu \mathrm{M}$; Tocris Bioscience), SR95531 (20 $\mu \mathrm{M}$; Tocris Bioscience), and CGP52432 (1 $\mu \mathrm{M}$; Tocris Bioscience). The R-type $\mathrm{Ca}^{2+}$ channel blocker SNX482 (Tocris Bioscience) was dissolved in water to a stock concentration of $100 \mu \mathrm{M}$ and stored at $-20^{\circ} \mathrm{C}$ until required. On the day of the experiment SNX482 was diluted in ACSF to a concentration of $1 \mu \mathrm{M}$ for local application. The high-affinityCa ${ }^{2+}$ chelatorBAPTA [1,2-bis(o-aminophenoxy)ethane$N, N, N^{\prime}, N^{\prime}$-tetraacetic acid] was added to the intracellular solution of the dendritic recording pipette at a concentration of $3 \mathrm{~mm}$ in some experiments. 
In vivo preparation and electrophysiology. Wistar rats (P30-P35) were anesthetized by inhalation of isoflurane (2\%) before they were injected with urethane (i.p., $1.5 \mathrm{~g} / \mathrm{kg}$ in a $20 \%$ solution in saline). The state of the animal was constantly checked throughout the experiment and if signs were observed that indicated a weakening of the aesthesia (e.g., toe pinching reflex), small doses of urethane were applied to keep the animal unconscious. The rat was then placed in a stereotactic frame (Narishige) and the body temperature maintained at $36^{\circ} \mathrm{C}$ with a temperature control and monitoring system (Sutter Instruments). A small craniotomy $(1 \times 1 \mathrm{~mm})$ was performed, $1.5 \mathrm{~mm}$ posterior to the bregma and $2 \mathrm{~mm}$ lateral to the midline to obtain access to the primary somatosensory cortex (Paxinos and Watson, 1998). To allow insertion of recording electrodes, a hole was made in the dura using a needle and the exposed cortical surface was perfused with normal rat ringer solution of the following composition (in mM): $135 \mathrm{NaCl}, 5.4 \mathrm{KCl}, 1.0 \mathrm{MgCl}_{2}, 1.8 \mathrm{CaCl}_{2}, 5$ HEPES, $\mathrm{pH}$ adjusted to 7.2 with $\mathrm{KOH}$. In vivo whole-cell recordings were obtained using the "blind" patch-clamp technique (Margrie et al., 2002). Electrodes were inserted into the brain at an oblique angle $\left(20-30^{\circ}\right)$ and lowered to a depth to record from layer 5 neurons. Sub-pial depths ranged from 700 to $950 \mu \mathrm{m}$, as estimated from the distance the micromanipulator (Sutter Instruments) had advanced, taking into account the angle of insertion. Whole-cell current-clamp recordings were made with a current-clamp amplifier (BVC-700A, Dagan) using glass pipettes (5-7 $\mathrm{M} \Omega$ tip resistance) filled with the same intracellular solution used for in vitro experiments. Biocytin $(0.2-0.5 \%)$ was added to the solution for visualization and histological identification of recorded neurons. At the end of electrophysiological recordings, rats were perfused transcardially with 0.1 м PBS followed by a solution of $4 \%$ paraformaldehyde (PFA). The brain was removed from the skull and kept in PFA overnight. Coronal slices (100 $\mu \mathrm{m}$ thick) of the somatosensory cortex were prepared and processed with the avidin-biotin peroxidase reaction (Vectastain ABC kit, Vector Laboratories) for visualization of cell morphology. Slices were mounted in Moviol and cells were visually inspected under an epifluorescence and confocal microscope (LSM 5 Pascal, Zeiss). Of a total of 16 in vivo recordings used for data analysis, four layer 5 pyramidal cells were successfully recovered.

In experiments where apamin was applied intracellularly, somatic patch pipettes were tip-filled with normal intracellular solution and then back-filled with internal solution containing apamin (100 nM). After break-in effects were observed after 5-15 min. Traces recorded immediately after break-in $(<2 \mathrm{~min})$ were used as control, whereas the impact of intracellular apamin on cellular properties used data obtained after $5 \mathrm{~min}$. All traces recorded from $2 \mathrm{~min}$ after break-in to $5 \mathrm{~min}$ were excluded from analysis. For experiments where apamin was applied to the cortical surface data were only accepted for analysis $>15 \mathrm{~min}$ after application.

Experimental design and statistical analysis. Both hemispheres from male and female animals were used for in vitro and in vivo experiments. Numbers in the Results section and figure legends describe the number of cells recorded. The number of animals and brain slices used is detailed in Table 1.

Data analysis was performed using AxoGraph $\mathrm{X}$ in combination with custom programs in MATLAB (MathWorks) as well as Microsoft Excel. Prism (GraphPad) was used for statistics and preparation of graphs. For paired data Wilcoxon's nonparametric matched pairs test or a paired $t$ test (if a Gaussian distribution could be assumed) was used to test statistical significance. If groups were split on two independent variables, twoway ANOVA was used to determine significance. For multiple datasets, Dunn's multiple-comparison test was performed to determine statistical significance. Comparison of cumulative probability distributions used the Kolmogorov-Smirnov test. Results are presented as average values \pm the SE. In the figures "NS" denotes not statistically significant, ${ }^{*} p<0.05$ and ${ }^{\star *} p<0.01$.

Passive membrane parameters, such as input resistance were measured by holding the cell at $-70 \mathrm{mV}$ and injecting multiple current steps, starting at $-30 \mathrm{pA}$ and incrementally increasing by $10 \mathrm{pA}$ to a maximum of $+50 \mathrm{pA}$. When assessing AP bursts, spikes were classified as part of an AP burst if two or more APs occurred with an interspike interval (ISI) $<18$ ms using an automated algorithm in MATLAB. Determination of
Table 1. Experimental statistics: number of animals, brain slices and cells for each individual experiment

\begin{tabular}{lccc}
\hline Experiment & Animals & Slices & Cells \\
\hline Effect of bath application of apamin (Fig. 1) & 8 & 10 & 10 \\
Effect of local apamin application (Fig. 2) & 9 & 10 & 10 \\
Effect of UCL1684 (Fig. 3) & 7 & 9 & 9 \\
Effect of NS309 (Fig. 4) & 7 & 8 & 8 \\
Effect of BAPTA (Fig. 5) & 5 & 5 & 5 \\
Effect of SNX282 (Fig. 6) & 5 & 5 & 5 \\
Effect on isolated calcium spikes (Fig. 7) & 5 & 5 & 5 \\
Effect of intracellular apamin in vitro (Fig. 9) & 5 & 13 & 13 \\
Effect of intracellular somatic apamin in vivo (Fig. 9) & 4 & N/A & 4 \\
Effect of apamin to the cortical surface in vivo (Fig. 10) & 12 & N/A & 12 \\
\hline
\end{tabular}

NA, Not applicable.

AP bursts was also checked manually to ensure accuracy. The mAHP amplitude at the soma was defined as the absolute value of the maximum negative voltage difference from AP threshold ( $s e t$ at $d V / d t=50 \mathrm{mV} / \mathrm{ms}$ ) in a time window $50 \mathrm{~ms}$ after AP onset. The dendritic AHP amplitude was defined as the absolute value of the maximum negative voltage difference from dendritic spike threshold (set at $d V / d t=40 \mathrm{mV} / \mathrm{ms}$ ) in a time window $100 \mathrm{~ms}$ after dendritic spike onset. In experiments on the critical frequency for dendritic spike generation (Larkum et al., 1999a) the integral of the dendritic membrane potential was determined during somatic AP trains at frequencies ranging from 50 to $200 \mathrm{~Hz}$ (in steps of $10 \mathrm{~Hz}$ ). The critical frequency was then defined as the frequency at which the derivative of the plot of AP frequency versus dendritic integral reached a maximum. To generate the normalized integral plots, data were normalized to the average integral above the critical frequency.

For analysis of UP and DOWN states in vivo the following procedure was implemented: A median filter over 500 sample points (100 ms) was applied to isolate the low-frequency components of voltage signals. This trace was then differentiated and a custom-built peak detection algorithm (in MATLAB, MathWorks) used to determine local maxima and minima identifying the onset and offset of UP and DOWN states. Visual inspection of unfiltered data indicated that these maxima and minima accurately identified transitions between UP and DOWN states, with maxima indicating the onset of an UP state and minima indicating the offset of an UP state (or the beginning of a DOWN state).

Modeling. Computer simulations were performed using the NEURON 7.4 simulation environment (Carnevale and Hines, 2006). A single compartment, conductance-based model of length $50 \mu \mathrm{m}$ and diameter 50 $\mu \mathrm{m}$ was used. The passive properties of the model were as follows: specific membrane resistance $10,000 \Omega \cdot \mathrm{cm}^{2}$, specific membrane capacitance $1 \mu \mathrm{F} / \mathrm{cm}^{2}$ and internal resistance $150 \Omega \cdot \mathrm{cm}$. All simulations were performed with the resting membrane potential set to $-55 \mathrm{mV}$ using an integration time constant of $25 \mu \mathrm{s}$. The model included two additional conductances: a low-threshold voltage-activated calcium channel (Migliore et al., 1995) and a calcium-dependent SK channel (Destexhe et al., 1993), modified to have an $\mathrm{EC}_{50}$ of $300 \mathrm{~nm}$, similar to experimental observations (Xia et al., 1998). The baseline intracellular calcium concentration was set at $150 \mathrm{~nm}$ and the rate of calcium removal was modeled by a single exponential decay with a time constant of $10 \mathrm{~ms}$, similar to experimental observations (Sabatini et al., 2002). The density of voltage-activated calcium channels and SK channels were varied between $10-200$ and $0-9 \mathrm{pS} / \mu \mathrm{m}^{2}$, respectively, as indicated in the Results. These values were chosen to replicate experimental data on the impact of SK channels on calcium spikes.

\section{Results}

\section{Impact of SK channels on neuronal excitability}

To investigate the effect of SK channels on somatic and dendritic excitability, dual recordings were established on the soma and the main apical dendrite of layer 5 pyramidal neurons $(600-800 \mu \mathrm{m}$ from the soma). Initially, SK channel activity was blocked at all locations by bath application of the SK channel blocker apamin $(100 \mathrm{nM})$. Consistent with previously published results, this led to 


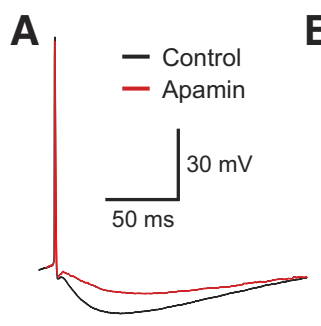

B
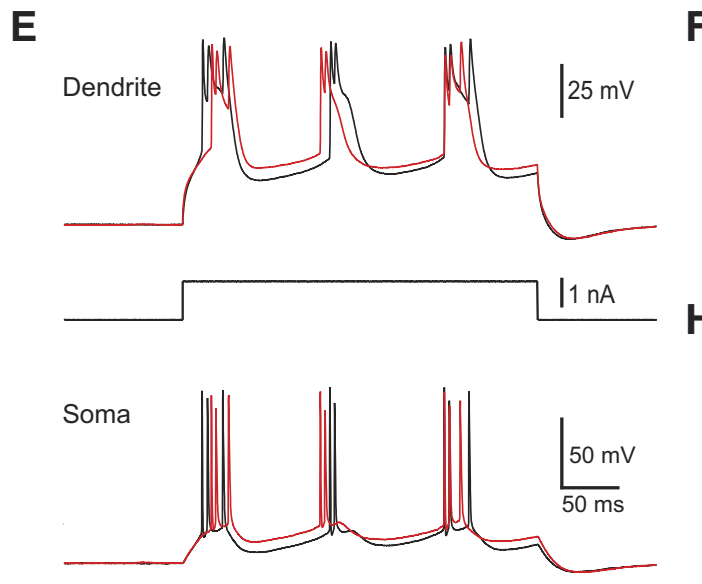

J

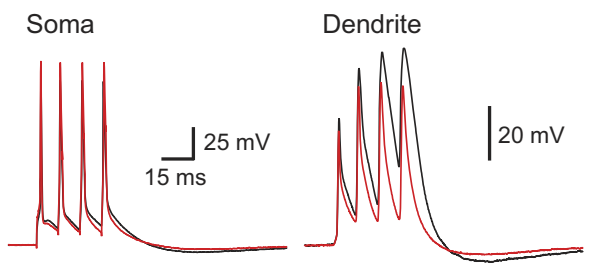

C

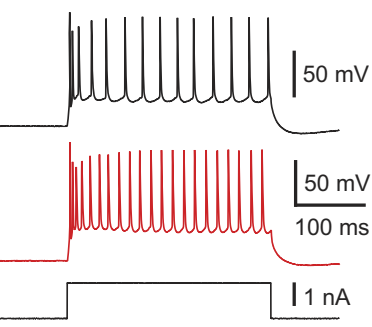

D

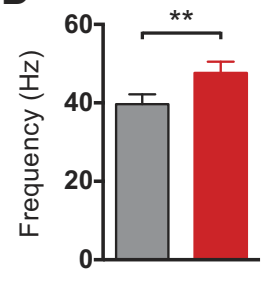

F

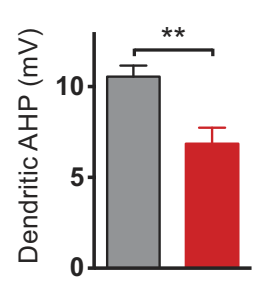

H

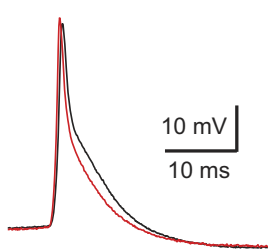

K

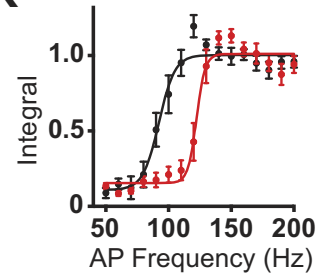

G
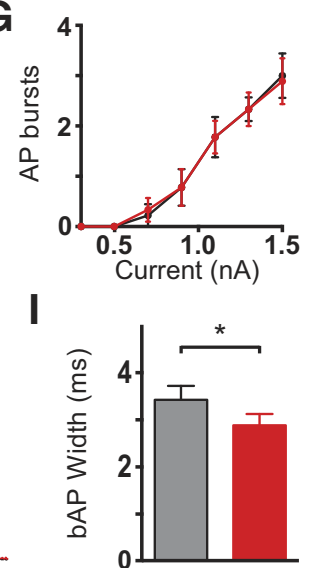

L

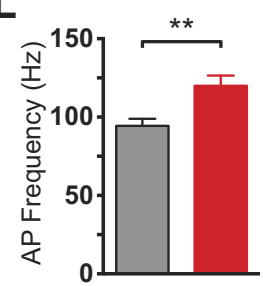

Figure 1. Effect of bath application of apamin on cell excitability. $A$, Somatic AP in control (black) and after bath application of apamin (red). B, Average amplitude of the somatic mAHP in control (black) and after bath application of apamin (red; $n=10$ ). C, Somatic response to a $500 \mathrm{~ms}$ current step (bottom) in control (black) and after bath application of apamin (red). $\boldsymbol{D}$, Average somatic firing frequency in response to $1.5 \mathrm{nA}$ current steps in control (black) and after bath application of apamin (red; $n=10)$. E, Somatic (bottom) and dendritic (top; $700 \mu \mathrm{m}$ from the soma) responses to a 300 ms dendritic current step (middle) in control (black) and after bath application of apamin (red; $100 \mathrm{~nm}$ ). $\boldsymbol{F}$, Average hyperpolarization after dendritic spikes (dendritic AHP) in control (black) and after bath application of apamin (red; $n=10)$. G. Average number of somatic AP bursts during dendritic current injections of different amplitude in control (black) and after bath application of apamin (red; $n=10)$. $\boldsymbol{H}$, bAP in control (black) and after bath application of apamin (red; $700 \mu \mathrm{m}$ from the soma). $\boldsymbol{I}$, Average bAP width at $25 \%$ amplitude in control (black) and after bath application of apamin (red; $n=10$ ). J, Somatic (left) and dendritic response (right; $600 \mu \mathrm{m}$ from the soma) to four somatic APs at the critical frequency (100 Hz) in control (black) and after bath application of apamin (red). $\boldsymbol{K}$, Averaged, normalized dendritic voltage integral during AP trains at the indicated AP frequencies in control (black) and after bath application of apamin (red; lines represent sigmoidal fits; $n=10$ ). $L$, Average critical frequency in control (black) and after bath application of apamin (red; $n=10$ ).

a reduction in the $\mathrm{mAHP}$ at the soma (Fig. $1 A, B ; p=0.002$ with Wilcoxon test) and an increase in AP firing (Fig. $1 C, D ; p=0.002$ with Wilcoxon test; $n=10$ ). To investigate the impact of bath application of apamin on dendritic excitability current steps were injected via the dendritic recording pipette, leading to the generation of dendritic calcium spikes and somatic burst firing (Fig. $1 E$; cf. Williams and Stuart, 1999). Similar to its effect on the mAHP at the soma (Fig. 1B), bath application of apamin reduced the hyperpolarization $50 \mathrm{~ms}$ after dendritic calcium spikes (Fig. $1 F ; p=0.0039$ with Wilcoxon test $n=10$ ), but had no impact on somatic AP burst firing during dendritic current injection (Fig. $1 G ; p=0.99$ with two-way ANOVA; $n=10$ ). Surprisingly, apamin also led to a decrease, rather than the expected increase, in the width of backpropagating APs (bAPs) at $25 \%$ of their peak amplitude (Fig. $1 H, I ; p=0.002$ with Wilcoxon test, $n=10$ ). Consistent with this unexpected effect on bAP width, the critical frequency of somatic AP firing required for generation of dendritic calcium spikes (Larkum et al., 1999a) was increased rather than decreased by bath application of apamin (Fig. $1 J-L ; p=$
0.0078 with Wilcoxon test; $n=10$ ). These observations indicate that blocking SK channels globally in cortical L5 neurons has complex effects on somatic and dendritic excitability, prompting further investigation by local manipulation of SK channels in somatic and dendritic compartments.

\section{Location-dependent effects of SK channels}

To determine the origin of SK channel effects seen during bath application of apamin, we locally applied apamin to the soma or the dendritic recording site near the apical nexus (Fig. 2A). Local application of apamin to the soma $(1 \mu \mathrm{M})$ decreased the somatic mAHP and increased AP firing (Fig. $2 B-E ; p=0.002$ and $p=$ 0.0038 , respectively, with Wilcoxon test; $n=10$ ), as observed during bath application of apamin (Fig. 1). In contrast, local application of apamin to the dendritic recording site did not affect the MAHP at the soma or AP firing during somatic current injection (Fig. $2 B-E ; p=0.85$ and $p=0.63$, respectively, with Wilcoxon test; $n=10$ ), indicating that dendritic apamin applications were restricted to dendritic regions. Unexpectedly, local 
A

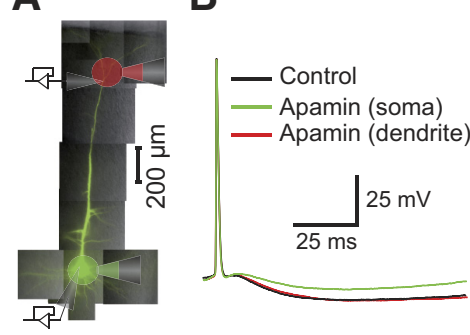

F
C

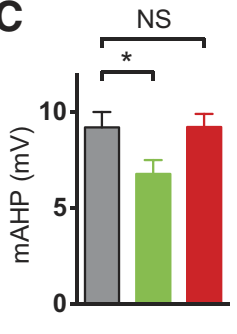

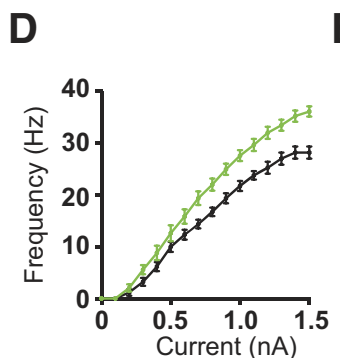
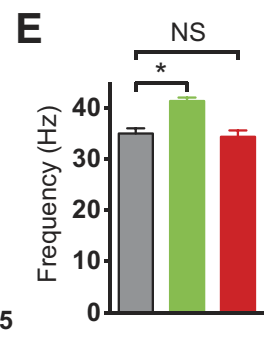

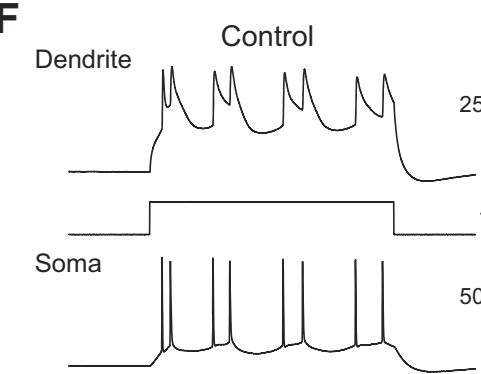

H

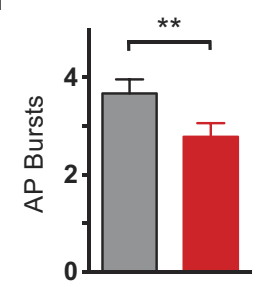

L

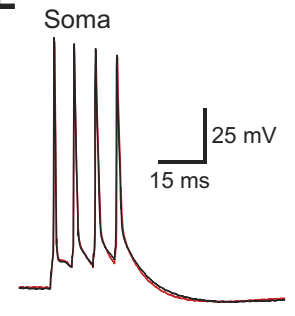

I

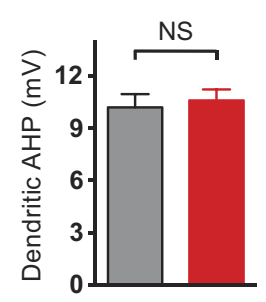

M
Apamin (dendrite)

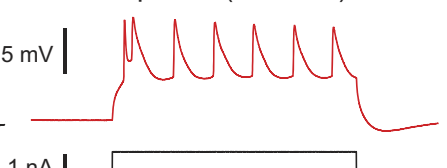

G

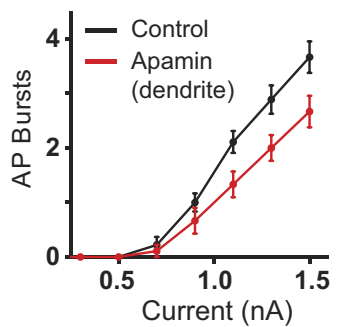

K J
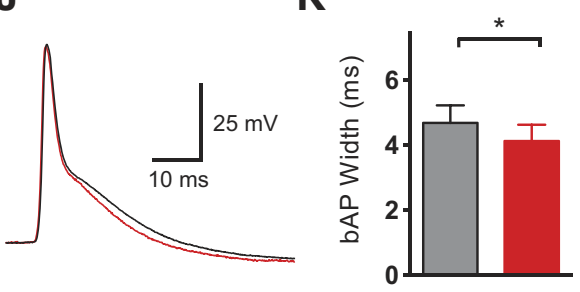

N
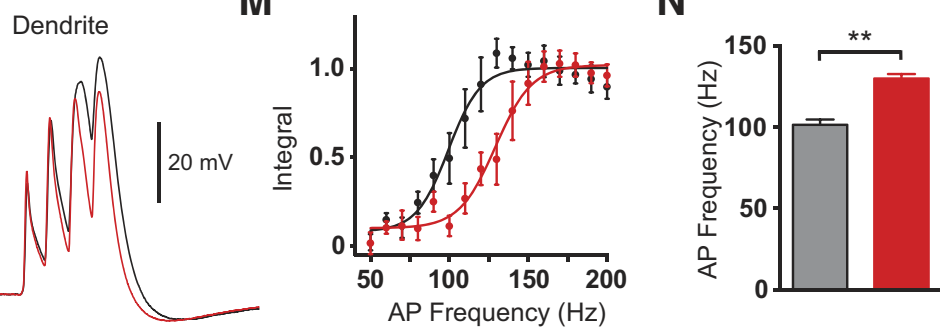

Figure 2. Effect of local apamin application on somatic and dendritic excitability. A, Schematic of the experimental setup showing an image of a layer 5 pyramidal neuron filled with the fluorescent dye AlexaFluor 594 together with somatic and dendritic recording and local application pipettes. B, Somatic APs in control (black) and after local somatic (green) or dendritic (red; 710 $\mu \mathrm{m}$ from the soma) apamin application. C, Average effect of local somatic (green) or dendritic (red) apamin application on the somatic mAHP ( $n=10$; black, control). D, Average F/l curves in control (black) and after somatic apamin application (green; $n=10)$. $\boldsymbol{E}$, Average AP firing frequency during somatic current injections (1.5nA) in control (black) and during local somatic (green) or dendritic (red) apamin application (green; $n=10$ ). $F$, Somatic (bottom) and dendritic (top; $710 \mu \mathrm{m}$ from the soma) responses to $300 \mathrm{~ms}$ dendritic current injections (middle) in control (black, left) and after local dendritic apamin (red, right). $\boldsymbol{G}$, Average number of AP bursts during dendritic current injections of different amplitude in control (black) and after local dendritic apamin (red; $n=10)$. $\boldsymbol{H}$, Average number of AP bursts during $1.5 \mathrm{nA}$ dendritic current steps in control (black) and after local dendritic apamin (red; $n=10$ ). $I$, Average effect of apamin on the hyperpolarization after dendritic calcium spikes (dendritic AHP) in control (black) and after local dendritic apamin (red). J, Backpropagating AP (bAP) before (black) and after local dendritic apamin (red; $670 \mu \mathrm{m}$ from the soma). $\boldsymbol{K}$, Average bAP width at $25 \%$ amplitude in control (black) and after local dendritic apamin (red; $n=10$ ). $L$, Somatic (left) and dendritic response (right; $710 \mu \mathrm{m}$ from the soma) to four somatic APs at the critical frequency $(130 \mathrm{~Hz}$ ) before (black) and after local dendritic apamin (red). $\boldsymbol{M}$, Averaged, normalized dendritic voltage integral during AP trains at the indicated frequencies in control (black) and after local dendritic apamin (red; lines represent sigmoidal fits; $n=10$ ). $N$, Average critical frequency in control (black) and after local dendritic apamin (red; $n=10$ ).

application of apamin to the dendritic recording site decreased, rather than increased, the generation of dendritic calcium spikes and somatic burst firing during dendritic current injection (Fig. $2 F-H ; p=0.0039$ with Wilcoxon test; $n=10$ ). This occurred in the absence of an effect of dendritic apamin on the hyperpolarization after dendritic calcium spikes (Fig. 2 I; $p=0.32$ with Wilcoxon test; $n=10$ ), which was reduced by somatic apamin application (data not shown; $p=0.027$ with Wilcoxon test; $n=$ $10)$. These data suggest that dendritic SK channels act to promote, rather than suppress, dendritic calcium spikes. Further, they indicate that the AHP after dendritic calcium spikes is mediated primarily by somatic SK channels. These different effects of dendritic and somatic SK channels appear to play off against each other during bath application of apamin, leading to no net change in AP burst firing (Fig. $1 G$ ). As seen during bath application of apamin (Fig. $1 H, I$ ), dendritic application of apamin decreased the duration of bAPs at $25 \%$ of their peak amplitude (Fig. $2 J, K ; p=0.0039$ with Wilcoxon test; $n=10$ ). As also seen during bath application (Fig. $1 J-L$ ), dendritic apamin applications lead to an increase in the critical frequency for evoking dendritic calcium spikes during high-frequency AP firing (Fig. $2 L-N ; p=$ 0.002 with Wilcoxon test; $n=10$ ). These data indicate that the impact of global SK channel block on bAP width at $25 \%$ of peak and critical frequency is due to block of dendritic SK channels. 
Similar effects to those described in Figure 2 were observed in the presence of glutamatergic and GABAergic receptor blockers $(n=$ 5; see Materials and Methods), ruling out the possibility that the inhibitory impact of apamin on dendritic excitability was due to modulation of presynaptic network activity. Together, these data suggest that somatic and dendritic SK channels have opposing effects on neuronal excitability, with dendritic SK channels paradoxically acting to enhance dendritic calcium spike generation and dendritic calcium electrogenesis.

To address potential nonspecific effects of apamin the experiments described above were repeated using local applications of the alternative SK channel blocker UCL1684. UCL1684 (100 $\mu \mathrm{M})$ application to the dendritic recording location in these experiments lead to a decrease in the generation of dendritic calcium spikes and somatic AP bursts during dendritic current injection (Fig. $3 A-C ; p=0.031$ with Wilcoxon test; $n=9$ ), as well as an increase in the critical frequency (Fig. $3 D, E ; p=0.031$ with Wilcoxon test; $n=9$ ). These observations are essentially identical to those observed during local dendritic application of apamin (Fig. 2).

We also investigated the impact of enhancing SK channel activity with NS309 (Strøbaek et al., 2000; Pedarzani et al., 2005). Local applications of NS309 $(100 \mu \mathrm{M})$ to the somatic and dendritic recording site had opposite effects on somatic and dendritic excitability to that seen with apamin and UCL1684. Local somatic application of NS309 enhanced the mAHP at the soma and reduced AP output during somatic current injection (Fig. $4 A-D$; $p=0.031$ and $p=0.025$, respectively, with Wilcoxon test; $n=8$ ). In contrast, local dendritic application of NS309 increased burst firing during dendritic current injection (Fig. $4 E, F ; n=8$ ) as well as the width of bAPs (Fig. $4 G, H ; p=0.016$ with Wilcoxon test; $n=8$ ), and decreased the critical frequency for generation of dendritic calcium spikes during high-frequency AP firing (Fig. $4 I-K ; p=0.031$ with Wilcoxon test; $n=8$ ).

Finally, to confirm that apamin was working via blocking a calcium-activated conductance, as would be expected if it was blocking SK channels, we investigated the impact of apamin on somatic and dendritic excitability after chelating intracellular calcium with BAPTA. BAPTA (3 mM) was added to the dendritic pipette solution and we waited at least $10 \mathrm{~min}$ after obtaining dendritic whole-cell recording for BAPTA to diffuse throughout the neuron. In the presence of BAPTA all effects of bath application of apamin on somatic and dendritic excitability were blocked (Fig. $5 ; n=5$ ). Following chelation of intracellular calcium with BAPTA bath application of apamin had no effect on somatic AP firing frequency and the somatic mAHP (Fig. $5 A-C$; $p=0.98$ and $p=0.81$, respectively, with Wilcoxon test; $n=5$ ). Bath application of apamin also had no effect on the number of AP bursts evoked by dendritic current injection (Fig. $5 D, E ; p=$ 0.5 with Wilcoxon test; $n=5$ ), the dendritic AHP amplitude (Fig. $5 F ; p=0.81$ with Wilcoxon test; $n=5$ ), the critical frequency for evoking dendritic calcium spikes during high-frequency AP firing (Fig. $5 G, H ; p=0.75$ with Wilcoxon test; $n=5$ ) or the width of bAPs (Fig. $5 I, J ; p=0.63$ with Wilcoxon test; $n=5$ ). These data confirm that the observed effects of apamin on somatic and dendritic excitability require intracellular calcium, as one would expect if they were mediated via SK channels. Furthermore, they exclude the possibility that apamin is acting on channels that do not require intracellular calcium for their activation, such as voltage-activated calcium channels. Together with the experiments using UCL1684 and NS309, these data strongly support the idea that the counter-intuitive inhibitory effects of apamin on dendritic excitability arise via block of SK channels.
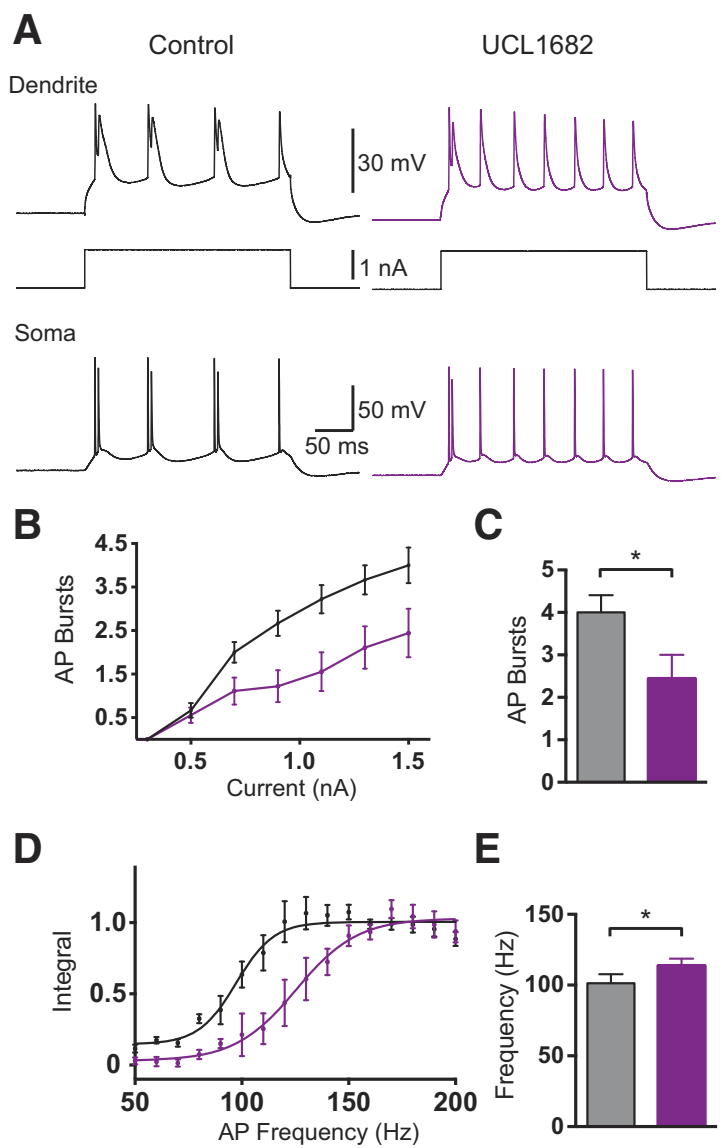

E

Figure 3. Effect of local dendritic application of UCL1684 on dendritic excitability.A, Somatic (bottom) and dendritic (top; $690 \mu \mathrm{m}$ from the soma) responses to dendritic current injections (middle) in control (black) and after local dendritic UCL1684 (purple). B, Average number of AP bursts during dendritic current injections of different amplitude (control: black; dendritic UCL1684: purple; dendritic recording $640-750 \mu \mathrm{m}$ from the soma; $n=9$ ). C, Average number of bursts during dendritic current injection (1.5 nA) in control (black) and after dendritic UCL1684 (purple; $n=9$ ). D, Average, normalized dendritic voltage integral during AP trains at different frequencies (lines represent sigmoidal fits, control: black, dendritic UCL1684: purple; dendritic recording $640-750 \mu \mathrm{m}$ from the soma; $n=9$ ). $\boldsymbol{E}$, Average critical frequency in control (black) and after dendritic UCL1684 (purple; $n=9$ ).

\section{Interaction of SK channels and R-type calcium channels}

Calcium-activated potassium channels can be activated by calcium influx through distinct calcium channel subtypes (Marrion and Tavalin, 1998; Pineda et al., 1998; Fakler and Adelman, 2008). To investigate the calcium source driving SK channel activation during dendritic calcium spikes we tested the impact of the R-type channel blocker SNX482 $(1 \mu \mathrm{M})$. Recent evidence indicates that calcium influx through R-type voltage-activated calcium channels drives SK channel activation in the dendrites and spines of cortical layer 5 pyramidal neurons during bAPs (Jones and Stuart, 2013). Furthermore, R-type channels are known to be activated during dendritic calcium spikes in these neurons (Pérez-Garci et al., 2013). In these experiments, SNX482 was locally applied to the apical dendritic recording site alone and subsequently in combination with apamin. Blocking dendritic R-type calcium channels with SNX482 decreased dendritic excitability leading to a reduction in burst firing (Fig. $6 A-C ; p=$ 0.0191 with Dunn's multiple-comparison test after a one-way ANOVA; $n=6$ ). Importantly, application of apamin in the presence of SNX482 had no additional impact on burst firing generated by dendritic current injection (Fig. $6 A-C ; p=0.999$ with 


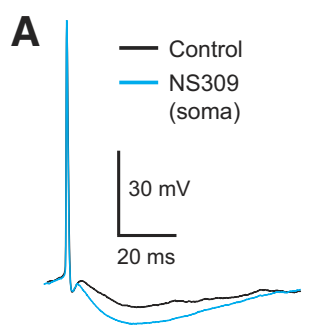

E

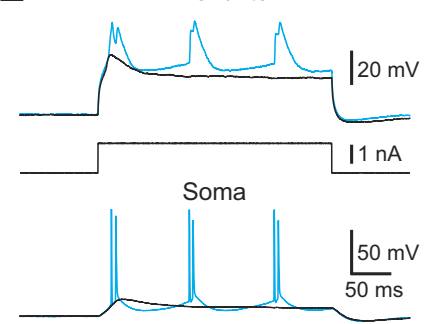

I

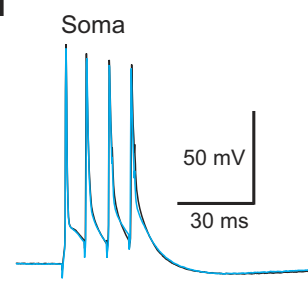

B

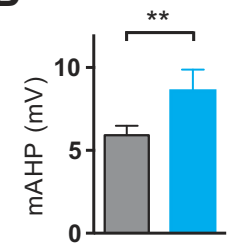

C

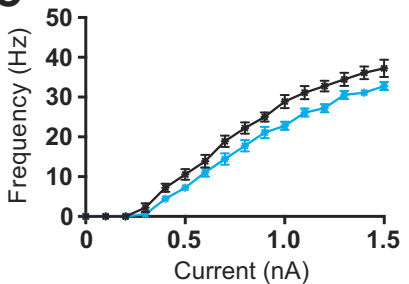

G
D

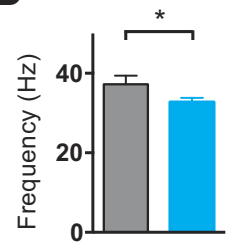

F

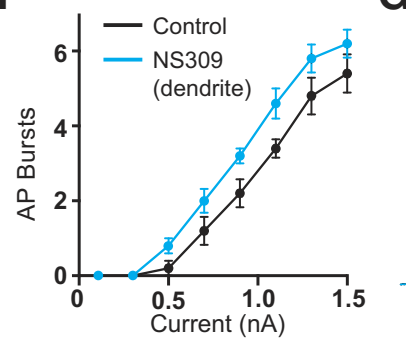

J

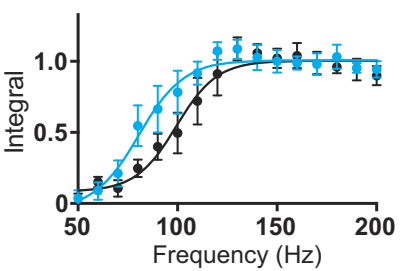

Dendrite

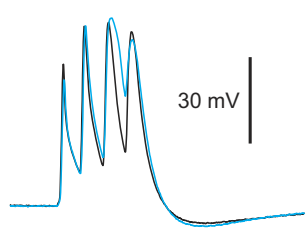

H

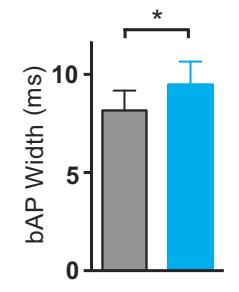

K
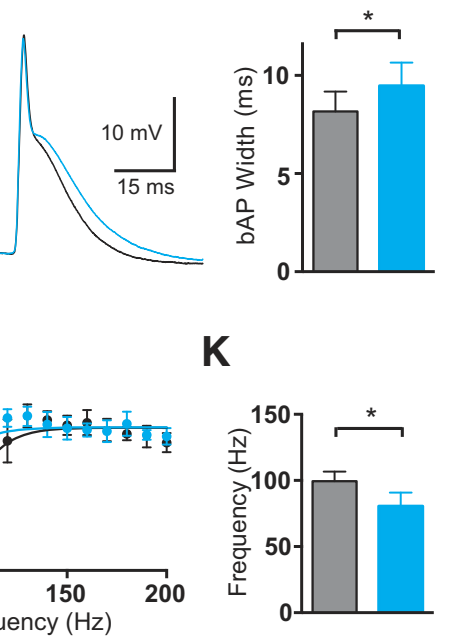

Figure 4. Effect of local NS309 application on somatic and dendritic excitability. A, Somatic AP in control (black) and after local somatic application of NS309 (blue). B, Average mAHP amplitude in control (black) and after local somatic NS309 (blue; $n=8$ ). C, Average F/l curve in control (black) and after local somatic NS309 (blue; $n=8$ ). $\boldsymbol{D}$, Average frequency of AP firing in control (black) and after local somatic NS309 (blue; $1.5 \mathrm{nA} \mathrm{somatic} \mathrm{current} \mathrm{injection;} n=8$ ). $E$, Somatic (lower) and dendritic (upper; $760 \mu \mathrm{m}$ from the soma) responses during dendritic current injection (middle) in control (black) and after local dendritic NS309 (blue). $F$, Average number of AP bursts observed during dendritic current injections of different amplitude in control (black) and after local dendritic NS309 (blue; $n=8$ ). G, bAP in control (black) and after local dendritic NS309 (blue; $700 \mu \mathrm{m}$ from the soma). $\boldsymbol{H}$, Average width of bAPs at $25 \%$ amplitude in control (black) and after local dendritic NS309 (blue; $n=8$ ). I, Somatic (left) and dendritic (right; $700 \mu \mathrm{m}$ from the soma) responses to four APs at the critical frequency (120 Hz) in control (black) and after local dendritic NS309 (blue). $\boldsymbol{J}$, Average, normalized dendritic voltage integral during AP trains of increasing frequency in control (black) and after local dendritic NS309 (blue; lines represent sigmoidal fits; $n=8$ ). $\boldsymbol{K}$, Average critical frequency in control (black) and after local dendritic NS309 (blue; $n=8$ ).

A

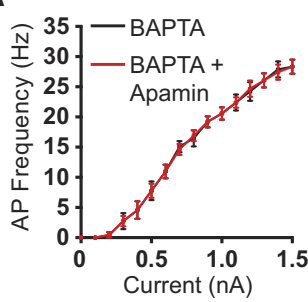

$\mathbf{F}$

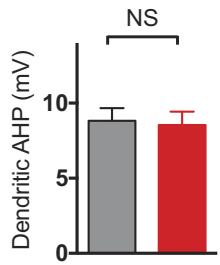

G
B
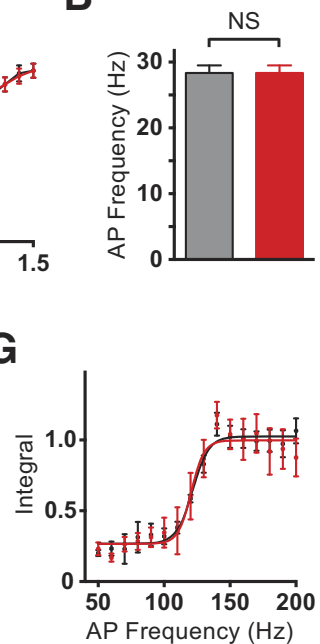

C

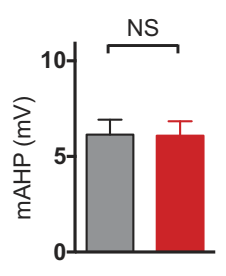

H

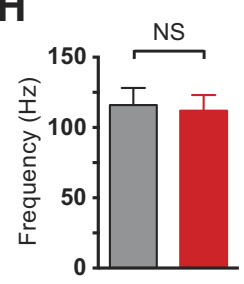

D

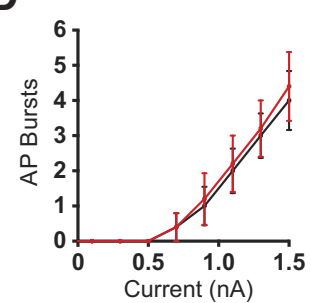

I

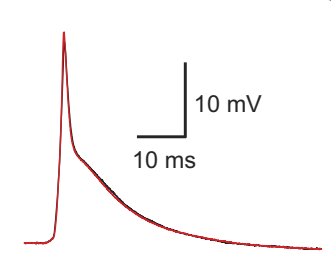

E

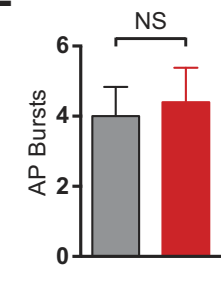

$\mathbf{J}$

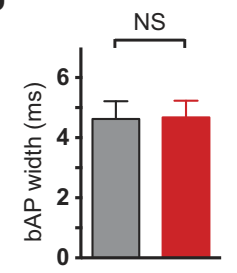

Figure 5. Effect of bath application of apamin after chelation of intracellular calcium with BAPTA. A, Average somatic $F / /$ curve before (black) and after (red; $n=5)$ bath application of apamin. $B$, Average AP frequency during somatic current injection ( $1.5 \mathrm{nA}$ ) in control (black) and after bath application of apamin (red; $n=5)$. C, Average somatic mAHP before (black) and after (red) bath application of apamin $(n=5)$. $\boldsymbol{D}$, Average number of AP bursts observed during dendritic current injection of varying intensity ( $600-800 \mu \mathrm{m}$ from the soma) in control (black) and after bath application of apamin (red; $n=5)$. $\boldsymbol{E}$, Average number of AP bursts during dendritic current injection ( $1.5 \mathrm{nA})$ in control (black) and after bath application of apamin (red; $n=5) . \boldsymbol{F}$, Average dendritic AHP before (black) and after (red) bath application of apamin $(n=5)$. G, Average, normalized dendritic voltage integral ( $600-740 \mu \mathrm{m}$ from the soma) during AP trains at different frequencies in control (black) and after bath application of apamin (red; lines represent sigmoidal fits; $n=5$ ). $\boldsymbol{H}$, Average critical frequency in control (black) and after bath application of apamin (red; $n=5$ ). $\boldsymbol{I}$, bAP in control (black) and after bath application of apamin (red). J, Average width of bAPs at $25 \%$ amplitude in control (black) and after bath application of apamin (red; $n=5$ ). 

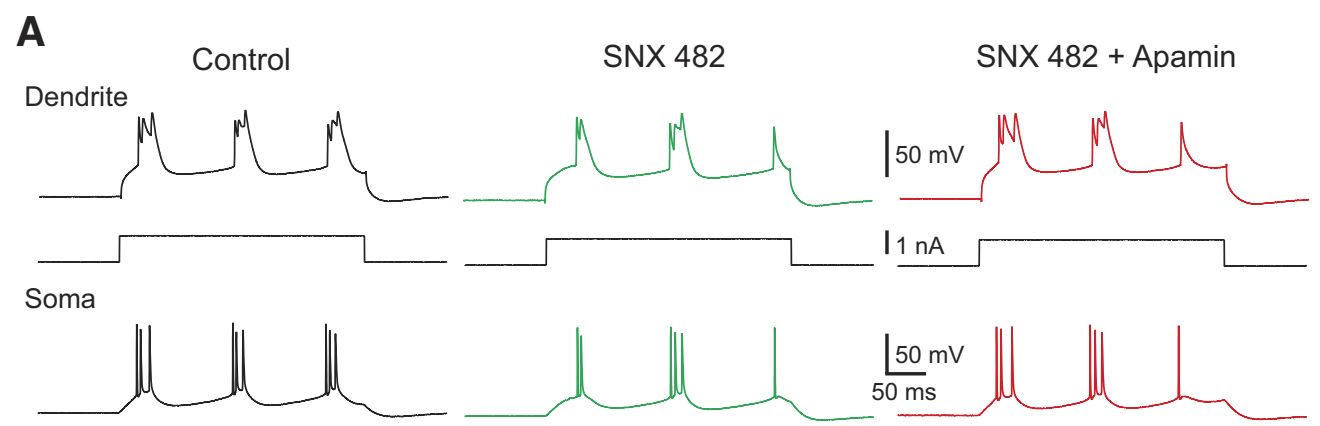

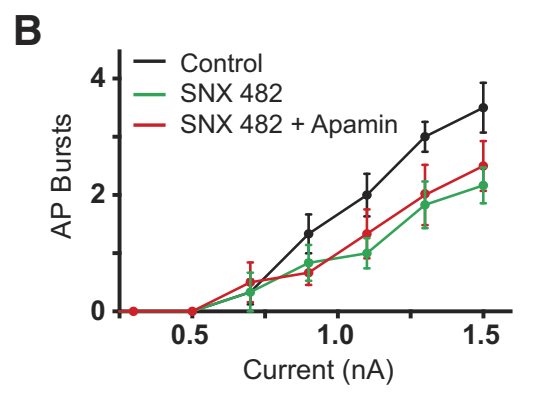

C

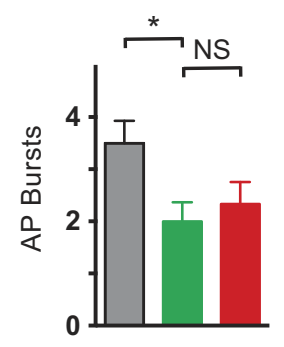

E
D
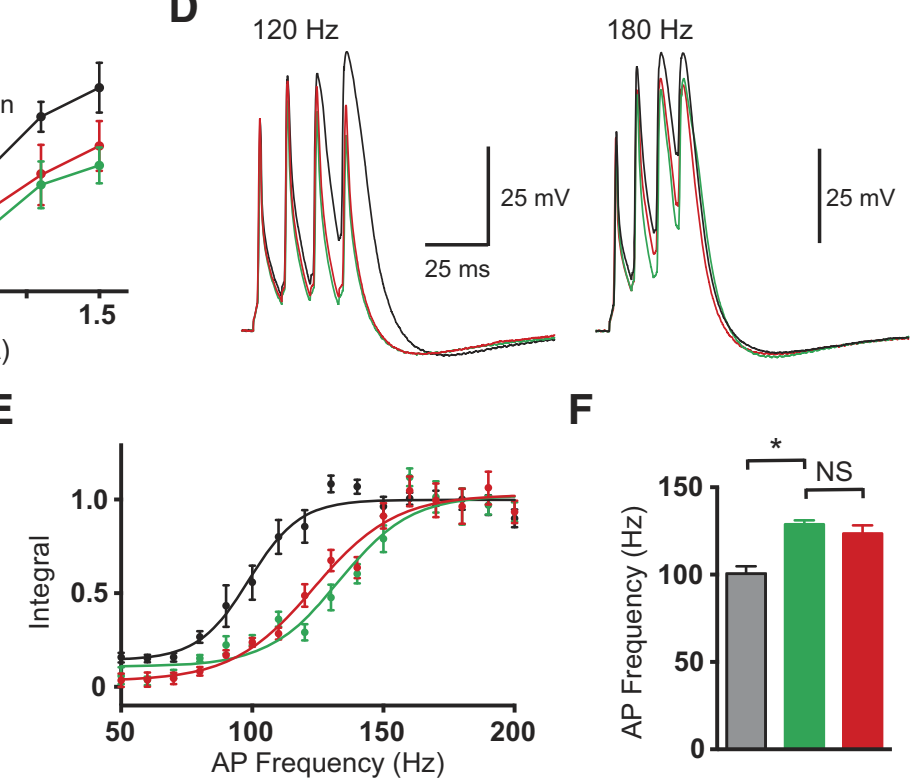

$\mathbf{F}$

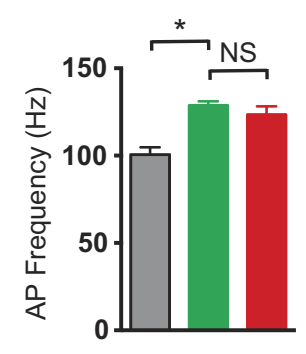

Figure 6. Effect R-type calcium channels. A, Dendritic (top; $680 \mu \mathrm{m}$ from the soma) and somatic (bottom) response to $300 \mathrm{~ms}$ dendritic current steps (middle) in control (black), after local dendritic SNX482 (green) and after local dendritic SNX482 plus apamin (red). B, Average number of AP bursts observed during dendritic current steps of varying intensity in control (black), after local dendritic SNX482 (green), and after local dendritic SNX482 plus apamin (red; $n=5$ ). C, Average number of AP bursts during a dendritic current step of $1.5 \mathrm{nA}$ in control (black), after local dendritic SNX482 (green), and after local dendritic SNX482 plus apamin (red; $n=5$ ). D, Dendritic responses ( $630 \mu \mathrm{m}$ from soma) to five somatic APs at $120 \mathrm{~Hz}$ (left) and $180 \mathrm{~Hz}$ (right) in control (black), after local dendritic SNX482 (green) and after local dendritic SNX482 plus apamin (red; $n=5$ ). $E$, Averaged, normalized dendritic voltage integral during AP trains at the indicated frequencies in control (black), after local dendritic SNX482 (green) and after local dendritic SNX482 plus apamin (red; lines represent sigmoidal fits; $n=5$ ). $\boldsymbol{F}$, Average critical frequency in control (black), after local dendritic SNX482 (green) and after local dendritic SNX482 plus apamin ( $\operatorname{red} n=5$ ).

Dunn's multiple-comparison test after a one-way ANOVA; $n=$ 6). Consistent with these observations, application of SNX482 alone also significantly increased the critical frequency, whereas adding apamin in the presence of SNX482 had no further impact (Fig. $6 D-F ; p=0.0032$ and $p>0.99$, respectively, with Dunn's multiple-comparison test after a one-way ANOVA with Wilcoxon test; $n=6$ ). These findings suggest that R-type calcium channels are the primary calcium source for SK channel activation during dendritic spikes. Furthermore, they indicate that block of R-type calcium channels both mimics and occludes the effect of SK channels on dendritic excitability.

\section{Impact of SK channels on dendritic excitability in the absence of APs}

To investigate the impact of SK channels on dendritic excitability in the absence of somatic APs, dendritic current injections at distal locations were used to evoke isolated dendritic spikes. Dual whole-cell recordings were established on the soma and the first-, second-, or third-order branches of tuft dendrites of layer 5 pyramidal neurons in these experiments (Fig. 7A; 700-870 $\mu \mathrm{m}$ from the soma). Apamin or NS309 were applied locally to the dendritic recording site to modulate SK channel activation. Den- dritic current injection via the dendritic recording pipette evoked local dendritic calcium spikes, which propagated to the soma but did not elicit somatic AP firing (Fig. 7 B, C). Modulating dendritic SK channel activity did not change the peak amplitude of isolated dendritic calcium spikes, but did change their duration. Local dendritic application of apamin decreased the half-width of isolated dendritic calcium spikes (Fig. $7 B, D ; p=0.039$ with Wilcoxon test, $n=5$ ), whereas local dendritic application of NS309 increased dendritic calcium spike half-width (Fig. $7 C, E ; p=$ 0.010 with Wilcoxon test, $n=5$ ). These results show that the impact of SK channels on dendritic excitability occurs locally and is not dependent on somatic AP firing.

Finally, we investigated the impact of local applications of apamin and NS309 on dendritic input resistance. In contrast to the absence of an impact of either of these compounds on somatic input resistance, apamin and NS309 had opposing effects on the input resistance of distal apical tuft dendrites. Blocking dendritic SK channels via local application of apamin to the distal dendritic tuft increased dendritic input resistance by on average $7 \%$, whereas local application of the SK channel enhancer NS309 decreased dendritic input resistance by $9 \%$ (Fig. $7 F$ ). When normalized to their respective controls, a significant difference was 

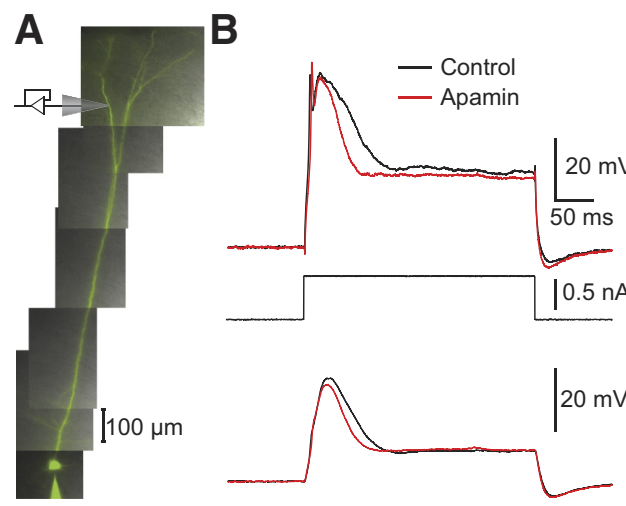

$\mathbf{F}$

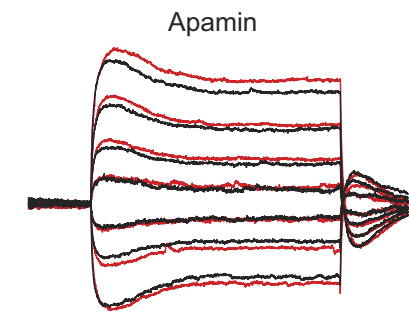

C
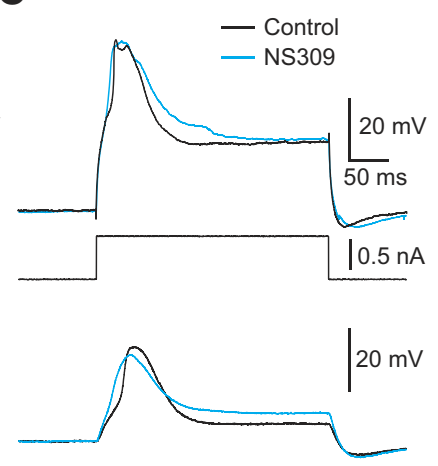

D

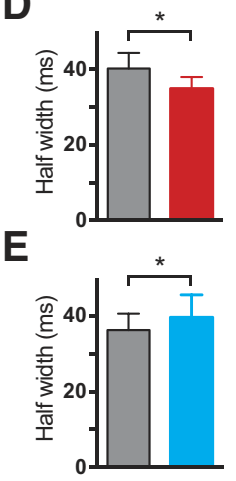

NS309

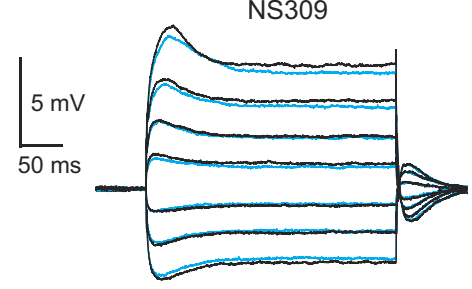

G

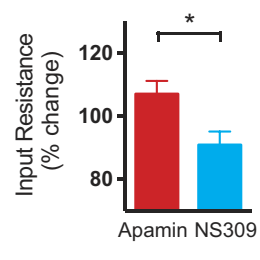

Figure 7. Effect of apamin and NS309 on isolated calcium spikes. A, Schematic of the experimental setup during simultaneous somatic and distal dendritic recording from a distal apical tuft dendrite. $B, C$, Somatic (bottom) and dendritic (top) responses during isolated dendritic calcium spikes evoked by dendritic current injection (middle) in control (black) and after local dendritic apamin ( $\boldsymbol{B}$; red; $860 \mu \mathrm{m}$ from the soma) or NS309 (C; blue; $830 \mu \mathrm{m}$ from the soma). $\boldsymbol{D}, \boldsymbol{E}$, Average dendritic calcium spike half-width in control (black) and apamin ( $\boldsymbol{D} ;$ red; $n=5)$ or NS309 (E; blue; $n=5)$. $\boldsymbol{F}$, Dendritic responses during low amplitude $(-0.3$ to $+0.4 \mathrm{nA})$ dendritic current injections in control (black) and after local dendritic apamin (left; red; $770 \mu \mathrm{m}$ from the soma) or NS309 (right; blue; $800 \mu \mathrm{m}$ from the soma). G, Average percentage change in input resistance at distal dendritic tuft recording sites normalized to control in apamin (red) and NS309 (blue; $n=5$ ).

observed on the impact of these SK channel modulators on input resistance in distal tuft dendrites (Fig. $7 G ; p=0.016$ with MannWhitney test; $n=5$ ). These data suggest that in contrast to SK channels at the soma, SK channels in the distal apical tuft of layer 5 pyramidal neurons may be active at resting membrane potentials.

\section{Modeling the impact of SK channels on calcium spikes}

The observation that dendritic SK channels are potentially active at resting membrane potentials may underlie their counterintuitive effects on dendritic excitability. To test this idea we built a single compartment model that included both SK channels and a voltage-activated calcium channel. In this model we set the SK channel $\mathrm{EC}_{50}$ to $300 \mathrm{~nm}$, as observed experimentally (Xia et al., 1998), and the internal calcium concentration to $150 \mathrm{nM}$. Increasing the SK channel density lead to hyperpolarization (Fig. 8A), as a result of constitutive SK channel activation. This effect occurred over SK channel densities that were physiologically realistic (1-10 $\left.\mathrm{pS} / \mu \mathrm{m}^{2}\right)$. Importantly, this SK-dependent hyperpolarization removed inactivation of voltage-activated calcium channels (Fig. $8 B$ ). This promoted the generation of calcium spikes in models where the density of voltage-activated calcium channels was high (Fig. 8C,D), whereas when the density of voltage-activated calcium channels was low SK channels inhibited calcium spike generation (Fig. $8 E, F$ ). By varying the SK and calcium channel densities we found that both excitatory and inhibitory effects of SK channels on calcium spike generation could be observed over a physiologically relevant range of SK channel densities (Fig. $8 G, H)$. In summary, these simulations indicate that constitutive activation of SK channels can remove inactivation of voltageactivated calcium channels, enhancing calcium spike generation under some conditions. This counter-intuitive effect of SK channels on calcium spike generation may explain the excitatory effect of dendritic SK channels seen experimentally.

\section{Impact of SK channels in vivo}

To investigate the effect of SK channels on dendritic integration in vivo we used intracellular application of apamin to block SK channels (Szente et al., 1988). Consistent with the idea that intracellular apamin blocks SK channels, application of apamin (100 $\mathrm{nM}$ ) via the somatic recording pipette had similar effects on the somatic mAHP and AP firing in vitro to extracellular apamin application. Inclusion of apamin (100 nM) in the somatic recording solution led to a $22 \%$ reduction in the mAHP ( $p=0.042$ with Wilcoxon test; $n=13$ ), with subsequent bath application of apamin having no additional effect (Fig. $9 A, B ; p=0.94$ with Wilcoxon test; $n=13$ ). AP firing frequency during somatic current steps was also increased after intracellular application of apamin ( $p=0.034$ with Wilcoxon test; $n=13)$, with subsequent bath application of apamin having no additional effect (Fig. $9 C, D ; p=0.89$ with Wilcoxon test; $n=13$ ). That intracellular application of apamin had similar effects on somatic excitability to extracellular application, combined with the fact that subsequent extracellular application of apamin had no additional impact on somatic excitability, indicates that intracellular apamin application is effective in blocking SK channels.

Next, we used this method to determine the impact of somatic SK channels on neuron excitability in vivo. Whole-cell recordings were obtained from neurons in layer 5 of somatosensory cortex in anesthetized rats. To obtain data as soon as possible after break-in we analyzed the impact of intracellular application of apamin (100 nM) on spontaneous APs evoked during UP states. The mAHP amplitude of these APs 15 min after break-in was decreased by $28 \%$ compared with that observed immediately $(<2$ min) after obtaining whole-cell recording (Fig. 9 E, F; $p=0.046$ with Wilcoxon test; $n=4)$. Furthermore, we found that the average number of spontaneous APs during UP states increased significantly 15 min after break-in compared with that obtained immediately ( $<2 \mathrm{~min}$ ) after obtaining whole-cell recording (Fig. 
A

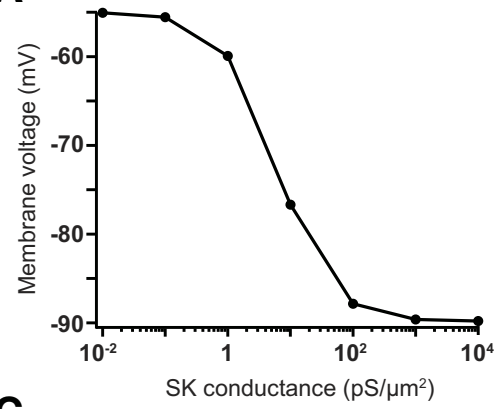

C

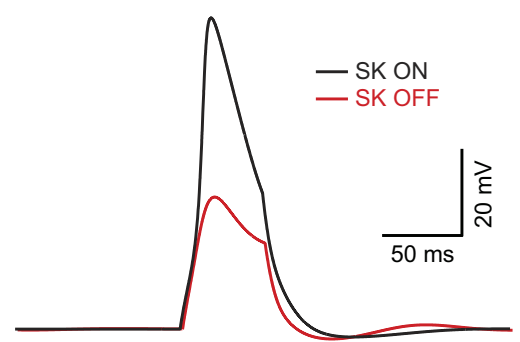

$\mathbf{E}$
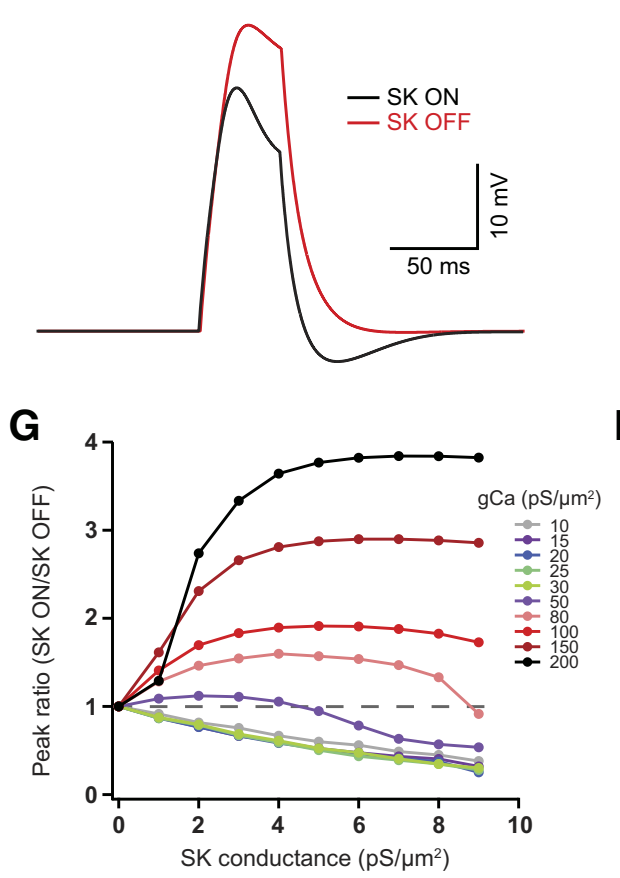

$\mathbf{F}$

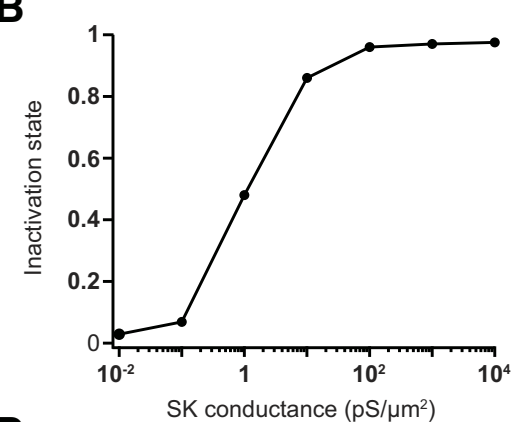

D
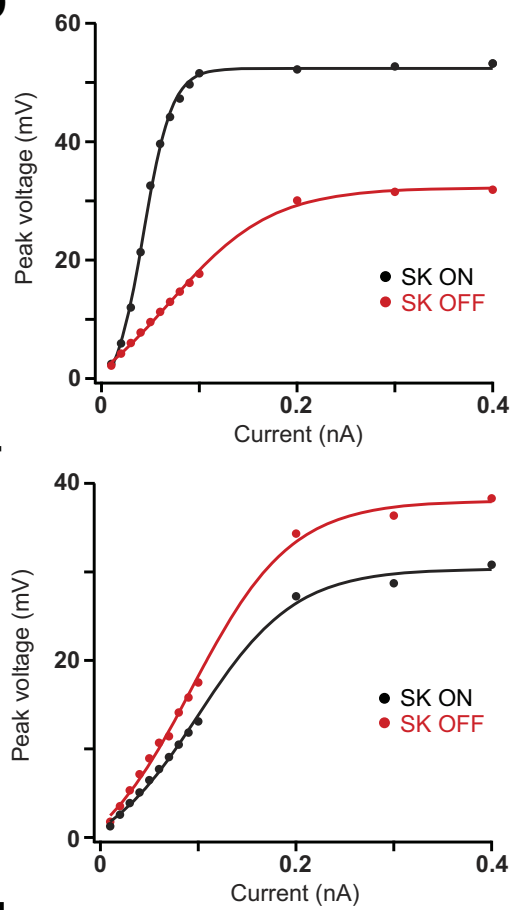

H

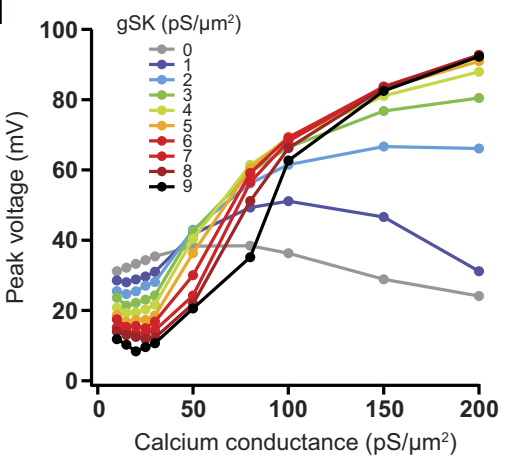

Figure 8. Modeling SK channel interactions with voltage-activated calcium channels. $\boldsymbol{A}$, Impact of varying the SK channel conductance (gSK) density on membrane potential. $\boldsymbol{B}$, Impact of varying gSK on availability of calcium channels. $\boldsymbol{C}, \boldsymbol{E}$, Calcium spikes evoked by brief current injections ( $50 \mathrm{~ms} ; 200 \mathrm{pA})$ in the presence (black) and absence (red) of SK channels $\left(2 \mathrm{pS} / \mu \mathrm{m}^{2}\right)$ in models with the voltage-activated calcium channel conductance density (gCa) set to $150 \mathrm{pS} / \mathrm{um}^{2}(\boldsymbol{C})$ or $15 \mathrm{pS} / \mathrm{um}^{2}$ (E). $\boldsymbol{D}, \boldsymbol{F}$ Amplitude of voltage responses to brief $(50 \mathrm{~ms})$ current injections of different amplitude in the presence (black) and absence (red) of SK channels $\left(2 \mathrm{pS} / \mu \mathrm{m}^{2}\right)$ in models with gCa set to $150 \mathrm{ps} / \mu \mathrm{m}^{2}(\boldsymbol{D})$ or $15 \mathrm{pS} / \mu \mathrm{m}^{2}(\boldsymbol{F})$. G, Ratio of calcium spike amplitude in models with SK channels present (SK ON) or absent (SK OFF) versus the gSK density in models with different gCa densities. Calcium spikes evoked by brief current injections ( $50 \mathrm{~ms} ; 200 \mathrm{pA}$ ). Ratios greater than one (dotted line) indicate an excitatory action of $\mathrm{SK}$ channels. $\boldsymbol{H}$, Amplitude of calcium spikes evoked by brief current injections $(50 \mathrm{~ms} ; 200 \mathrm{pA})$ versus gCa in models with different $\mathrm{gSK}$ densities.

$9 G, H ; p=0.039$ with Wilcoxon test; $n=4)$. These results are essentially identical to our findings during internal SK channel block in vitro (Fig. 9A-D) and are consistent with our findings during somatic SK channel block (Fig. $2 A-E$ ). We conclude that as observed in vitro, the primary role of somatic SK channels in vivo is to reduce the overall AP output of layer 5 pyramidal neurons.

We next determined the impact of dendritic SK channels on neuronal output in vivo. To block dendritic SK channels apamin was applied to the surface of the cortex above the recorded cell at a high concentration (10 $\mu \mathrm{M} ; 10$ times that used during local dendritic applications in vitro). We anticipate that these applications of apamin will preferentially block SK channels in the upper cortical layers, which contain the apical tuft dendrites of layer 5 pyramidal neurons. To test whether these extracellular applications influence network activity we analyzed their impact on subthreshold membrane potential changes during UP and DOWN states. Membrane potential changes during UP and DOWN states were well fit with double Gaussian distributions (Fig. $10 A-C)$. Application of apamin to the cortical surface had no impact on the halfwidth (Fig. $10 D ; p=0.65$ during UP states and $p=0.45$ during DOWN states with Wilcoxon test; $n=12$ ) or mean of Gaussian fits to subthreshold membrane potential changes during UP and DOWN states (Fig. 10E; $p=0.68$ during UP states and $p=0.64$ during DOWN states with Wilcoxon test; $n=12$ ). This analysis suggests that apamin applications to the cortical surface do not have a significant impact on subthreshold membrane potential changes and therefore network activity.

We next evaluated the impact of apamin application to the cortical surface on AP output. In contrast to the effect of intracellular SK channel block, application of apamin to the cortical surface did not lead to a change in the somatic MAHP (Fig. 10F; $p=0.93$ with Wilcoxon test; $n=12)$ or the number of APs generated during UP states (Fig. 10G; $p=0.95$ with Wilcoxon test; $n=12$ ). These data suggest that apamin did not diffuse to layer 5 and block somatic SK channels. Consistent with the idea that blocking dendritic SK channels reduces high-frequency AP burst firing, as seen in vitro (Fig. $2 \mathrm{~F}-\mathrm{H}$ ), cumulative probability plots of the ISI during UP states indicated that application of apamin to the cortical surface shifted the ISI distribution to longer ISIs (Fig. 10H; $p=0.042$ with Kolmogorov-Smirnov test; $n=12$ ). In addition, when looking at the distribution of APs during UP states an effect of SK channel block in the upper cortical layers became apparent. Under control conditions, there is a bias for APs to occur during the initial $300 \mathrm{~ms}$ of UP states, which is absent following application of apamin to upper cortical layers (Fig. 10I). To quantify 
A
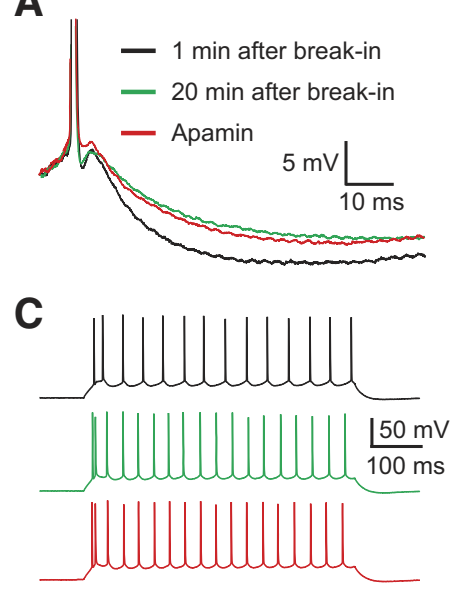

E

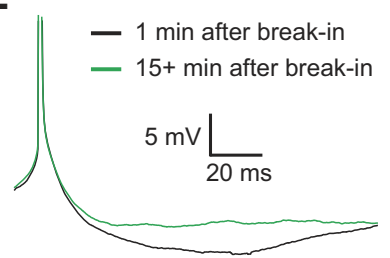

G

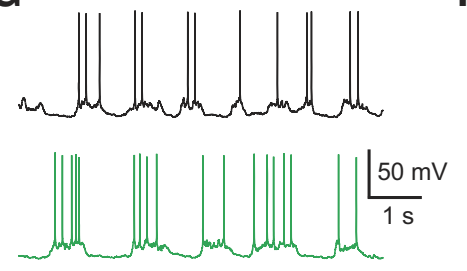

B

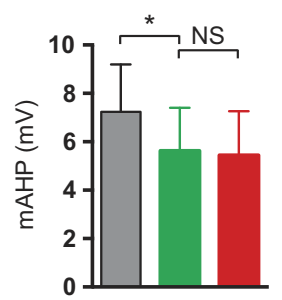

D

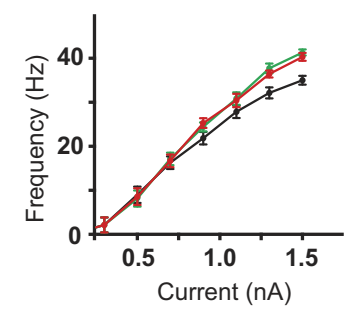

F

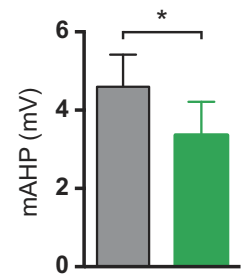

H

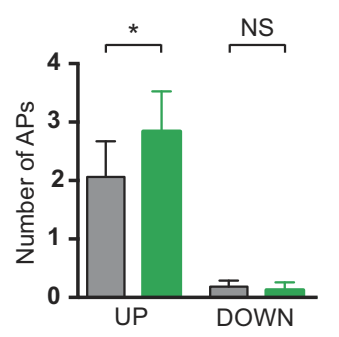

Figure 9. Effect of intracellular somatic apamin in vitro and in vivo. A, APs recorded immediately after break-in (black), 20 min after break-in (green) and after bath application of apamin (red) in vitro. B, Average mAHP amplitude immediately after break-in (black), 20 min after break-in (green) and after bath application of apamin (red) in vitro $(n=13)$. C, AP firing in response to $500 \mathrm{~ms}, 1.5 \mathrm{nA}$ somatic current injections immediately after break-in (black), 20 min after break in (green) and after bath application of apamin (red) in vitro. D, Average F/I curves immediately after break-in (black), 20 min after break in (green) and after bath application of apamin (red) in vitro $(n=13)$. $E$, Average of 50 aligned, spontaneous APs immediately after break-in (black) and 15 min after break-in (green) in vivo. $\boldsymbol{F}$, Average mAHP amplitude immediately after break-in (black) and 15 min after break-in (green) in vivo $(n=4)$. G, AP firing during UP and DOWN states immediately after break-in (black) and 15 min after break-in (green) in vivo. $\boldsymbol{H}$, Average number of APs during UP and DOWN states immediately after break-in (black) and 15 min after break-in (green) in vivo $(n=4)$.

this bias, the ratio of the average number of APs during the first $300 \mathrm{~ms}$ of an UP state was compared with that during the subsequent $300 \mathrm{~ms}$. Under control conditions this ratio is $\sim 1.5$, indicating that on average $50 \%$ more APs occur within the first 300 $\mathrm{ms}$ of an UP state compared with the subsequent $300 \mathrm{~ms}$. In contrast, after apamin application to the cortical surface this ratio drops to $\sim 1$ (Fig. $10 \mathrm{~J} ; p=0.026$ with Wilcoxon test; $n=12$ ). In summary, these data indicate that block of distal dendritic SK channels in vivo increases ISI and abolishes the clustering of APs at the beginning of UP states. This effect appears to be dendritic as it occurs in the absence of a change in the mAHP at the soma or the overall number of APs evoked during UP states (Fig. 10F, G), and is consistent with the impact of local dendritic SK channel block observed in vitro (Fig. 2).

\section{Discussion}

The results presented indicate that somatic and dendritic SK channels have opposing effects on the excitability of cortical layer 5 pyramidal neurons. While somatic SK channels contribute to the mAHP and limit AP output, dendritic SK channels enhance dendritic spike generation and AP burst firing. Comparable effects of somatic and dendritic SK channels were observed in vivo. Local manipulation of dendritic SK channel activity revealed that dendritic SK channels increase dendritic excitability by promoting the occurrence of dendritic calcium spikes. Consistent with this idea, blocking dendritic SK channels decreased the width of bAPs and increased the critical frequency for dendritic spike generation during high-frequency AP firing (Fig. 2), whereas enhancing dendritic SK channel activation increased bAP width and decreased the critical frequency (Fig. 4). These counterintuitive results indicate that dendritic SK channels are likely to promote, rather than inhibit, the impact of distal synaptic input on AP output (Larkum et al., 1999b, 2009; Williams and Stuart, 2002).

Although the inhibitory impact of somatic SK channels on neuronal excitability is well established (Schwindt et al., 1988a, 1992; Lorenzon and Foehring, 1992; Hirschberg et al., 1999; Jones and Stuart, 2013), what underlies the excitatory impact of dendritic SK channels? Calcium influx during dendritic calcium spikes and bAPs was expected to promote activation of dendritic SK channels, repolarizing the dendritic membrane potential and curtailing these regenerative events rather than enhancing them. This more traditional role of calcium-activated potassium conductances is observed during activation of dendritic BK channels, which have been shown to decrease dendritic calcium spike generation and duration in both cortical and hippocampal pyramidal neurons (Golding et al., 1999; Benhassine and Berger, 2009; Bock and Stuart, 2016a). Although the impact of SK channels on dendritic calcium spikes in pyramidal neurons has not previously been tested, in CA1 hippocampal pyramidal neurons SK channel activation limits the duration of plateau potentials generated by dendritic glutamate uncaging (Cai et al., 2004). In contrast, our findings in cortical layer 5 pyramidal neurons indicate that dendritic SK channels act to increase the width of dendritic calcium spikes as well as bAPs.

We hypothesize that this counterintuitive excitatory action of SK channels on dendritic excitability arises due to constitutive activation of SK channels, which acts to hyperpolarize the dendritic membrane potential. As we show in a single compartment model (Fig. 8), hyperpolarization mediated by constitutive activation of SK channels reduces inactivation of dendritic calcium channels and can enhance the generation of calcium spikes. Although apamin had no detectable impact on resting membrane properties at the soma, in recordings from apical tuft branches blocking SK channels increased distal dendritic input resistance, whereas enhancing SK channel activation had the opposite effect (Fig. 7). These data support the idea that SK channels are constitutively active at distal dendritic locations in layer 5 pyramidal neurons. Our inability to detect an effect of apamin on dendritic membrane potential presumably indicates that the hyperpolarization generated by constitutive SK channel activation occurs at a site distal to the dendritic recording location or within microdomains, possibly within dendritic spines. Constitutive activation of SK channels may also explain the excitatory impact SK channels have on NMDA spikes in basal dendrites of layer 5 pyramidal neurons, where they act to decrease the threshold for NMDA spike generation (Bock and Stuart, 2016b). 
A
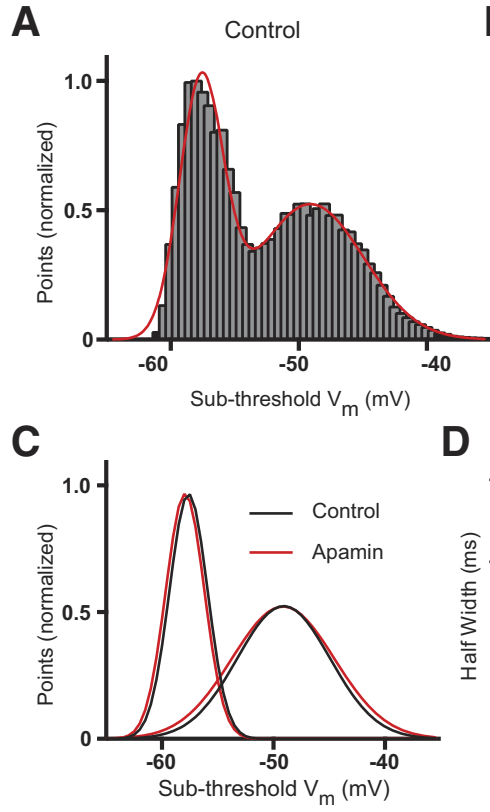

D

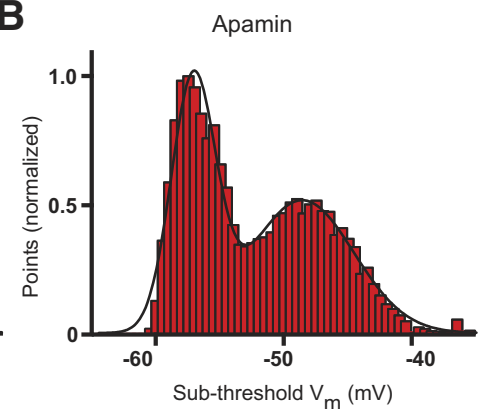

E
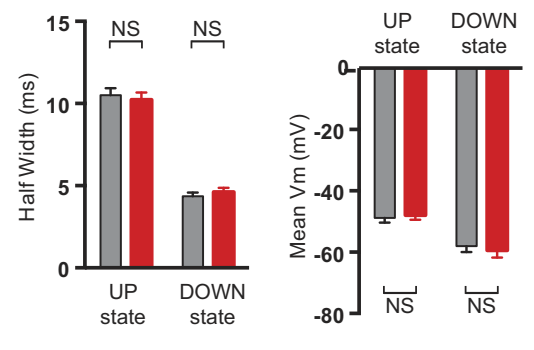

$\mathbf{F}$

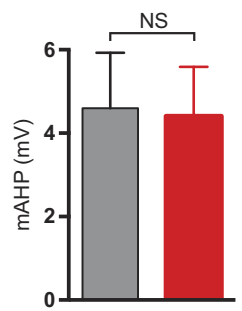

G

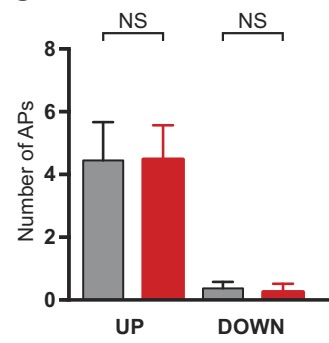

H

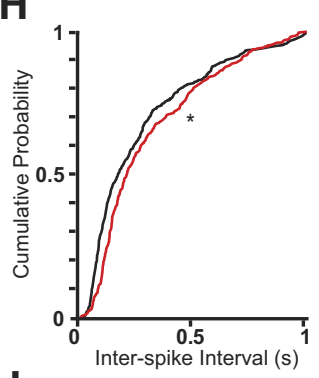

I
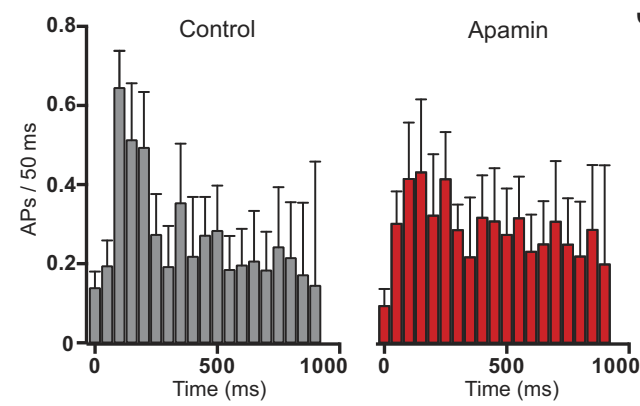

$\mathrm{J}$

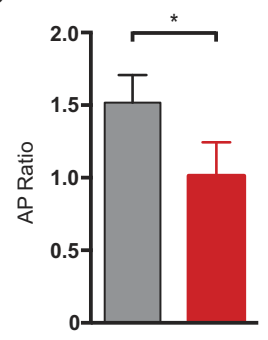

Figure 10. Effect of apamin application to the cortical surface in vivo. A, All points histograms of the median filtered voltage during $5 \mathrm{~min}$ of recording under control conditions. Binned with a resolution of $0.5 \mathrm{mV} / \mathrm{bin}$. The smooth line indicates a double Gaussian fit. $\boldsymbol{B}$, Same as $\boldsymbol{A}$ after the application of apamin to the cortical surface. $\boldsymbol{C}$, Overlaid Gaussian fits during UP and DOWN states based on the fits to the data in $\boldsymbol{A}$ and $\boldsymbol{B}$ (black, control; red, apamin to the cortical surface). $\boldsymbol{D}, \boldsymbol{E}$, Average half-width (D) and mean $(\boldsymbol{E})$ of Gaussian distributions during UP and DOWN states (black, control; red, apamin to the cortical surface; $n=12$ ). $\boldsymbol{F}$, Average mAHP amplitude before (black) and after (red) application of apamin to the cortical surface $(n=12)$. G, Average number of APs during UP and DOWN states in control (black) and after apamin application to the cortical surface (red; $\boldsymbol{n}=12$ ). $\boldsymbol{H}$, Cumulative probability distribution of ISI during UP states before (black) and after (red) application of apamin to the cortical surface $(n=12)$. I, Average number of APs during UP states in control (black) and after application of apamin to the cortical surface (red; $n=12$ ). Data binned in $50 \mathrm{~ms}$ epochs. J, Average ratio of the number of APs during the first $300 \mathrm{~ms}$ of UP states versus that during the subsequent $300 \mathrm{~ms}$ (black, control; red, apamin; $n=12$ ).

Differences in constitutive activation of somatic compared with dendritic SK channels could arise for a number of reasons. For example, the affinity of somatic SK channels for calcium may be lower than that of dendritic SK channels. Another possibility is that the resting calcium concentration may be higher at dendritic compared with somatic locations. Dendrites of cortical layer 5 pyramidal neurons are more depolarized than the soma (Stuart et al., 1997), which may lead to calcium influx through dendritic

voltage-activated calcium channels at resting membrane potentials (Magee et al., 1995). This could drive constitutive activation of SK channels in dendrites. A third possibility is that the coupling of somatic and dendritic SK channels to calcium influx through voltage-activated calcium channels may be different. Consistent with this idea, work in cortical layer 5 pyramidal neurons indicates that dendritic SK channels are more effectively coupled to calcium channels than somatic SK channels (Jones and Stuart, 2013).

The capacity of dendritic SK channels to promote the generation of dendritic calcium spikes was dependent on activation of R-type calcium channels (Fig. 6). This observation is consistent with an earlier finding showing that the reduction in NMDA spike threshold by SK channels is also dependent on activation of R-type calcium channels (Bock and Stuart, 2016b). Other work indicates that SK channels in dendritic spines form microdomains with R-type calcium channels during EPSPs (Bloodgood and Sabatini, 2008) and bAPs (Jones and Stuart, 2013). Together, these data suggest that microdomains formed by dendritic SK and R-type calcium channels are likely to be essential for their capacity to regulate dendritic excitability, and that this interaction may occur in spines. Finally, it is worth noting that although previous work indicates that the R-type channel blocker SNX482 can also block A-type (Kv4.2/3) potassium channels (Kimm and Bean, 2014), this is unlikely to have occurred in our experiments. Block of dendritic A-type potassium channels would be expected to increase dendritic excitability (Hoffman et al., 1997; Stuart and Häusser, 2001). In contrast, application of SNX482 decreased dendritic excitability, reducing burst firing and increasing the critical frequency (Fig. 6).

\section{SK channels in vivo}

By blocking SK channels internally we show that the reduction in cellular excitability caused by somatic SK channels has an impact on AP output in vivo (Fig. 9). These data suggest that somatic SK channels will act to modulate the impact of incoming sensory information by reducing the gain of cortical layer 5 pyramidal neurons. In this respect, somatic SK channels of layer 5 pyramidal neurons exhibit a similar function to that described to SK channels in other brain regions, like the hippocampus (McKay et al., 2012) and number of subcortical nuclei (Herrik et al., 2010; Gui et al., 2012; Alix et al., 2014). In contrast, application of apamin to the cortical surface indicated that dendritic SK channels promote shorter ISIs and cluster AP output to the initial phase of UP states (Fig. 10). These observations in vivo are consistent with our find- 
ings during block of dendritic SK channels in vitro, and provide further support for the idea that dendritic SK channels act to enhance dendritic spike generation and associated somatic burst firing. Distal apamin applications in vivo did not influence the somatic $\mathrm{mAHP}$, indicating they were restricted to dendritic locations. Although we cannot rule out the possibility that applications of apamin to the cortical surface influenced network activity, the finding that they did not influence subthreshold membrane potential changes during UP and DOWN states makes this unlikely. Overall, our findings are consistent with other work showing that changes in dendritic excitability have an impact on AP generation in vivo (Xu et al., 2012; Grienberger et al., 2014; Palmer et al., 2014). Finally, it is worth noting that the anesthetic used in our in vivo experiments (urethane) has been shown to suppress the generation of dendritic spikes in vitro (Potez and Larkum, 2008). As a result, we may have underestimated the excitatory impact of dendritic SK channels in vivo, which may be even greater in the awake, un-anaesthetized state.

From a functional perspective, layer 5 neurons receive direct sensory input from thalamocortical connections mainly onto their basal dendrites, whereas the majority of inputs onto distal apical dendrites are comprised of top-down corticocortical connections (Petreanu et al., 2009). Because dendritic spikes can act as coincidence detectors during synchronized input to distal apical dendrites (Larkum et al., 1999b, 2009; Williams and Stuart, 2002; Berger and Lüscher, 2003), they may play a role in functions such as memory formation (Gambino et al., 2014). If so, dendritic SK channels may act to enhance synaptic plasticity in cortical pyramidal neurons. Consistent with this idea, reducing SK channel activation in prefrontal cortex deceases rather than increases performance in working memory tasks (Brennan et al., 2008).

In conclusion, we describe a paradoxical excitatory impact of dendritic SK channels on neuronal output, which acts to enhance the generation of dendritic calcium spikes and promote somatic burst firing in vitro. More indirect experiments in vivo suggest a similar role of dendritic SK channels when neurons are embedded in their normal network. In contrast, somatic SK channels act to suppress AP output both in vitro and in vivo. Understanding how these opposing excitatory and inhibitory actions of SK channels influence learning and memory, as well as other tasks, will require targeted modulation of somatic and dendritic SK channels in awake, behaving animals. Further work will also be required to unravel the precise cellular mechanism(s) underlying the paradoxical excitatory effects of SK channels on dendritic excitability.

\section{References}

Alix P, Venkatesan K, Scuvée-Moreau J, Massotte L, Nguyen Trung ML, Cornil CA, Seutin V (2014) Mechanism of the medium-duration afterhyperpolarization in rat serotonergic neurons. Eur J Neurosci 39:186196.

Amitai Y, Friedman A, Connors BW, Gutnick MJ (1993) Regenerative activity in apical dendrites of pyramidal cells in neocortex. Cereb Cortex 3:26-38.

Benhassine N, Berger T (2009) Large-conductance calcium-dependent potassium channels prevent dendritic excitability in neocortical pyramidal neurons. Pflugers Arch 457:1133-1145.

Berger T, Lüscher HR (2003) Timing and precision of spike initiation in layer V pyramidal cells of the rat somatosensory cortex. Cereb Cortex 13:274-281.

Bittner KC, Grienberger C, Vaidya SP, Milstein AD, Macklin JJ, Suh J, Tonegawa S, Magee JC (2015) Conjunctive input processing drives feature selectivity in hippocampal CA1 neurons. Nat Neurosci 18:1133-1142.

Bloodgood BL, Sabatini BL (2007) Nonlinear regulation of unitary synaptic signals by $\mathrm{CaV}_{2.3}$ voltage-sensitive calcium channels located in dendritic spines. Neuron 53:249-260.

Bloodgood BL, Sabatini BL (2008) Regulation of synaptic signalling by postsynaptic, non-glutamate receptor ion channels. J Physiol 586:1475-1480.

Bock T, Stuart GJ (2016a) The impact of BK channels on cellular excitability depends on their subcellular location. Front Cell Neurosci 10:206

Bock T, Stuart GJ (2016b) Impact of calcium-activated potassium channels on NMDA spikes in cortical layer 5 pyramidal neurons. J Neurophysiol 115:1740-1748.

Brennan AR, Dolinsky B, Vu MA, Stanley M, Yeckel MF, Arnsten AF (2008) Blockade of IP3-mediated SK channel signaling in the rat medial prefrontal cortex improves spatial working memory. Learn Mem 15:93-96.

Cai X, Liang CW, Muralidharan S, Kao JP, Tang CM, Thompson SM (2004) Unique roles of SK and Kv4.2 potassium channels in dendritic integration. Neuron 44:351-364.

Carnevale NT, Hines ML (2006) The neuron book. Cambridge, UK: Cambridge.

Destexhe A, Babloyantz A, Sejnowski TJ (1993) Ionic mechanisms for intrinsic slow oscillations in thalamic relay neurons. Biophys J 65:15381552.

Faber ES, Delaney AJ, Sah P (2005) SK channels regulate excitatory synaptic transmission and plasticity in the lateral amygdala. Nat Neurosci 8: $635-641$.

Fakler B, Adelman JP (2008) Control of $\mathrm{K}_{\mathrm{Ca}}$ channels by calcium nano/ microdomains. Neuron 59:873-881.

Gambino F, Pagès S, Kehayas V, Baptista D, Tatti R, Carleton A, Holtmaat A (2014) Sensory-evoked LTP driven by dendritic plateau potentials in vivo. Nature 515:116-119.

Golding NL, Jung HY, Mickus T, Spruston N (1999) Dendritic calcium spike initiation and repolarization are controlled by distinct potassium channel subtypes in CA1 pyramidal neurons. J Neurosci 19:8789-8798.

Grienberger C, Chen X, Konnerth A (2014) NMDA receptor-dependent multidendrite $\mathrm{Ca}^{2+}$ spikes required for hippocampal burst firing in vivo. Neuron 81:1274-1281.

Gu N, Hu H, Vervaeke K, Storm JF (2008) SK (KCa2) channels do not control somatic excitability in CA1 pyramidal neurons but can be activated by dendritic excitatory synapses and regulate their impact. J Neurophysiol 100:2589-2604.

Gui L, LaGrange LP, Larson RA, Gu M, Zhu J, Chen QH (2012) Role of small conductance calcium-activated potassium channels expressed in PVN in regulating sympathetic nerve activity and arterial blood pressure in rats. Am J Physiol Regul Integr Comp Physiol 303:R301-R310.

Herrik KF, Christophersen P, Shepard PD (2010) Pharmacological modulation of the gating properties of small conductance $\mathrm{Ca}^{2+}$-activated $\mathrm{K}+$ channels alters the firing pattern of dopamine neurons in vivo. J Neurophysiol 104:1726-1735.

Hirschberg B, Maylie J, Adelman JP, Marrion NV (1999) Gating properties of single SK channels in hippocampal CA1 pyramidal neurons. Biophys J 77:1905-1913.

Hoffman DA, Magee JC, Colbert CM, Johnston D (1997) K+ channel regulation of signal propagation in dendrites of hippocampal pyramidal neurons. Nature 387:869-875.

Jones SL, Stuart GJ (2013) Different calcium sources control somatic versus dendritic SK channel activation during action potentials. J Neurosci 33: 19396-19405

Jones SL, To MS, Stuart GJ (2017) Dendritic small conductance calciumactivated potassium channels activated by action potentials suppress EPSPs and gate spike-timing dependent synaptic plasticity. Elife 6:e30333.

Kimm T, Bean BP (2014) Inhibition of A-type potassium current by the peptide toxin SNX-482. J Neurosci 34:9182-9189.

Larkum ME, Kaiser KM, Sakmann B (1999a) Calcium electrogenesis in distal apical dendrites of layer 5 pyramidal cells at a critical frequency of back-propagating action potentials. Proc Natl Acad Sci U S A 96: $14600-14604$.

Larkum ME, Zhu JJ, Sakmann B (1999b) A new cellular mechanism for coupling inputs arriving at different cortical layers. Nature 398:338-341.

Larkum ME, Nevian T, Sandler M, Polsky A, Schiller J (2009) Synaptic integration in tuft dendrites of layer 5 pyramidal neurons: a new unifying principle. Science 325:756-760.

Lorenzon NM, Foehring RC (1992) Relationship between repetitive firing and afterhyperpolarizations in human neocortical neurons. J Neurophysiol 67:350-363. 
Magee JC, Carruth M (1999) Dendritic voltage-gated ion channels regulate the action potential firing mode of hippocampal CAl pyramidal neurons. J Neurophysiol 82:1895-1901.

Magee JC, Christofi G, Miyakawa H, Christie B, Lasser-Ross N, Johnston D (1995) Subthreshold synaptic activation of voltage-gated $\mathrm{Ca}^{2+}$ channels mediates a localized $\mathrm{Ca}^{2+}$ influx into the dendrites of hippocampal pyramidal neurons. J Neurophysiol 74:1335-1342.

Manita S, Suzuki T, Homma C, Matsumoto T, Odagawa M, Yamada K, Ota K, Matsubara C, Inutsuka A, Sato M, Ohkura M, Yamanaka A, Yanagawa Y, Nakai J, Hayashi Y, Larkum ME, Murayama M (2015) A top-down cortical circuit for accurate sensory perception. Neuron 86:1304-1316.

Margrie TW, Brecht M, Sakmann B (2002) In vivo, low-resistance, wholecell recordings from neurons in the anaesthetized and awake mammalian brain. Pflugers Arch 444:491-498.

Marrion NV, Tavalin SJ (1998) Selective activation of $\mathrm{Ca}^{2+}$-activated K+ channels by co-localized $\mathrm{Ca}^{2+}$ channels in hippocampal neurons. Nature 395:900-905.

McKay BM, Oh MM, Galvez R, Burgdorf J, Kroes RA, Weiss C, Adelman JP, Moskal JR, Disterhoft JF (2012) Increasing SK2 channel activity impairs associative learning. J Neurophysiol 108:863-870.

Migliore M, Cook EP, Jaffe DB, Turner DA, Johnston D (1995) Computer simulations of morphologically reconstructed CA3 hippocampal neurons. J Neurophysiol 73:1157-1168.

Ngo-Anh TJ, Bloodgood BL, Lin M, Sabatini BL, Maylie J, Adelman JP (2005) SK channels and NMDA receptors form a $\mathrm{Ca}^{2+}$-mediated feedback loop in dendritic spines. Nat Neurosci 8:642-649.

Palmer LM, Shai AS, Reeve JE, Anderson HL, Paulsen O, Larkum ME (2014) NMDA spikes enhance action potential generation during sensory input. Nat Neurosci 17:383-390.

Paxinos G, Watson C (1998) The rat brain in stereotaxic coordinates, Ed 4. San Diego: Academic.

Pedarzani P, McCutcheon JE, Rogge G, Jensen BS, Christophersen P, Hougaard C, Strøbaek D, Stocker M (2005) Specific enhancement of SK channel activity selectively potentiates the afterhyperpolarizing current I(AHP) and modulates the firing properties of hippocampal pyramidal neurons. J Biol Chem 280:41404-41411.

Pérez-Garci E, Larkum ME, Nevian T (2013) Inhibition of dendritic $\mathrm{Ca}^{2+}$ spikes by $\mathrm{GABAB}$ receptors in cortical pyramidal neurons is mediated by a direct $\mathrm{G}_{\mathrm{i} / \mathrm{o}}-\beta \gamma$-subunit interaction with $\mathrm{Ca}_{\mathrm{v}} 1$ channels. J Physiol 591:1599-1612.

Petreanu L, Mao T, Sternson SM, Svoboda K (2009) The subcellular organization of neocortical excitatory connections. Nature 457:1142-1145.

Pineda JC, Waters RS, Foehring RC (1998) Specificity in the interaction of HVA Ca ${ }^{2+}$ channel types with $\mathrm{Ca}^{2+}$-dependent AHPs and firing behavior in neocortical pyramidal neurons. J Neurophysiol 79:2522-2534.

Potez S, Larkum ME (2008) Effect of common anesthetics on dendritic properties in layer 5 neocortical pyramidal neurons. J Neurophysiol 99:1394-1407.
Regehr W, Kehoe JS, Ascher P, Armstrong C (1993) Synaptically triggered action potentials in dendrites. Neuron 11:145-151.

Sabatini BL, Oertner TG, Svoboda K (2002) The life cycle of $\mathrm{Ca}^{2+}$ ions in dendritic spines. Neuron 33:439-452.

Sah P, McLachlan EM (1992) Potassium currents contributing to action potential repolarization and the afterhyperpolarization in rat vagal motoneurons. J Neurophysiol 68:1834-1841.

Schiller J, Schiller Y, Stuart G, Sakmann B (1997) Calcium action potentials restricted to distal apical dendrites of rat neocortical pyramidal neurons. J Physiol 505:605-616.

Schiller J, Major G, Koester HJ, Schiller Y (2000) NMDA spikes in basal dendrites of cortical pyramidal neurons. Nature 404:285-289.

Schwindt PC, Spain WJ, Foehring RC, Chubb MC, Crill WE (1988a) Slow conductances in neurons from cat sensorimotor cortex in vitro and their role in slow excitability changes. J Neurophysiol 59:450-467.

Schwindt PC, Spain WJ, Foehring RC, Stafstrom CE, Chubb MC, Crill WE (1988b) Multiple potassium conductances and their functions in neurons from cat sensorimotor cortex in vitro. J Neurophysiol 59:424-449.

Schwindt PC, Spain WJ, Crill WE (1992) Calcium-dependent potassium currents in neurons from cat sensorimotor cortex. J Neurophysiol 67: $216-226$.

Strøbaek D, Jørgensen TD, Christophersen P, Ahring PK, Olesen SP (2000) Pharmacological characterization of small-conductance $\mathrm{Ca}^{2+}$-activated $\mathrm{K}^{+}$channels stably expressed in HEK 293 cells. Br J Pharmacol 129: 991-999.

Stuart GJ, Häusser M (2001) Dendritic coincidence detection of EPSPs and action potentials. Nat Neurosci 4:63-71.

Stuart GJ, Sakmann B (1994) Active propagation of somatic action potentials into neocortical pyramidal cell dendrites. Nature 367:69-72.

Stuart G, Schiller J, Sakmann B (1997) Action potential initiation and propagation in rat neocortical pyramidal neurons. J Physiol 505:617-632.

Szente MB, Baranyi A, Woody CD (1988) Intracellular injection of apamin reduces a slow potassium current mediating afterhyperpolarizations and IPSPs in neocortical neurons of cats. Brain Res 461:64-74.

Takahashi N, Oertner TG, Hegemann P, Larkum ME (2016) Active cortical dendrites modulate perception. Science 354:1587-1590.

Williams SR, Stuart GJ (1999) Mechanisms and consequences of action potential burst firing in rat neocortical pyramidal neurons. J Physiol 521: 467-482.

Williams SR, Stuart GJ (2002) Dependence of EPSP efficacy on synapse location in neocortical pyramidal neurons. Science 295:1907-1910.

Xia XM, Fakler B, Rivard A, Wayman G, Johnson-Pais T, Keen JE, Ishii T, Hirschberg B, Bond CT, Lutsenko S, Maylie J, Adelman JP (1998) Mechanism of calcium gating in small-conductance calcium-activated potassium channels. Nature 395:503-507.

Xu NL, Harnett MT, Williams SR, Huber D, O'Connor DH, Svoboda K, Magee JC (2012) Nonlinear dendritic integration of sensory and motor input during an active sensing task. Nature 492:247-251. 